\title{
In search of exogenous feature-based attention
}

\author{
Ian Donovan ${ }^{1} \cdot$ Ying Joey Zhou ${ }^{1} \cdot$ Marisa Carrasco ${ }^{1,2}$
}

Published online: 17 July 2019

(C) The Psychonomic Society, Inc. 2019

\begin{abstract}
Visual attention prioritizes the processing of sensory information at specific spatial locations (spatial attention; SA) or with specific feature values (feature-based attention; FBA). SA is well characterized in terms of behavior, brain activity, and temporal dynamics - for both top-down (endogenous) and bottom-up (exogenous) spatial orienting. FBA has been thoroughly studied in terms of top-down endogenous orienting, but much less is known about the potential of bottom-up exogenous influences of FBA. Here, in four experiments, we adapted a procedure used in two previous studies that reported exogenous FBA effects, with the goal of replicating and expanding on these findings, especially regarding its temporal dynamics. Unlike the two previous studies, we did not find significant effects of exogenous FBA. This was true (1) whether accuracy or RT was prioritized as the main measure, (2) with precues presented peripherally or centrally, (3) with cue-to-stimulus ISIs of varying durations, (4) with four or eight possible target locations, (5) at different meridians, (6) with either brief or long stimulus presentations, (7) and with either fixation contingent or noncontingent stimulus displays. In the last experiment, a postexperiment participant questionnaire indicated that only a small subset of participants, who mistakenly believed the irrelevant color of the precue indicated which stimulus was the target, exhibited benefits for valid exogenous FBA precues. Overall, we conclude that with the protocol used in the studies reporting exogenous FBA, the exogenous stimulus-driven influence of FBA is elusive at best, and that FBA is primarily a top-down, goal-driven process.
\end{abstract}

Keywords Attention: Selective $\cdot$ Precuing $\cdot$ Visual perception

We cannot possibly process all available sensory information, and thus a subset of the environment must be selectively attended to and prioritized. Searching for an object of interest requires focusing on specific aspects of a visual scene while ignoring others. Visual attention is central to this process: It enhances perception and performance when stimuli appear at an attended location or possess an attended feature value. We can control the selection of visual information via endogenous attention, a sustained top-down voluntary process that allocates attention according to our internal goals and intentions. Additionally, bottom-up or stimulus-driven information can automatically direct exogenous attention, a reflexive process

Marisa Carrasco

marisa.carrasco@nyu.edu

1 Department of Psychology, New York University, New York, NY, USA

2 Center for Neural Science, New York University, New York, NY, USA that transiently prioritizes a subset of visual information following external stimulation (for reviews, see Carrasco, 2011, 2014; Carrasco \& Barbot, 2015).

Covert spatial attention (SA), the prioritization of information at discrete spatial locations without moving the eyes, is well characterized in terms of both exogenous and endogenous attention, and they are associated with partially overlapping cortical networks (Corbetta \& Shulman, 2002; Dugué, Merriam, Heeger, \& Carrasco, 2017). Their effects on visual perception are often similar but can differ (e.g., Barbot \& Carrasco, 2017; Barbot, Landy, \& Carrasco, 2012; Jigo \& Carrasco, 2018; Yeshurun, Montagna, \& Carrasco, 2008). Several studies have characterized the time courses of these two types of spatial attentional selection (e.g., Cheal, Lyon, \& Hubbard, 1991; Hein, Rolke, \& Ulrich, 2006; Ling \& Carrasco, 2006; Liu, Stevens, \& Carrasco, 2007b; Müller \& Rabbitt, 1989; Nakayama \& Mackeben, 1989; Remington, Johnston, \& Yantis, 1992). Specifically, exogenous SA results in benefits at the cued location after 100-120 ms, and quickly drops off afterwards; the enhancement may be followed by a 
period of suppression ("inhibition of return") before returning to baseline. Endogenous SA is deployed more slowly, taking about $300 \mathrm{~ms}$, but can be sustained at will. The understanding of the temporal dynamics of exogenous and endogenous attention is vital to characterizing their neural substrates, influences on perception, and how to best manipulate them to take advantage of their costs and benefits on performance.

Feature-based attention (FBA), the selection of particular visual feature values across the visual field, is also highly useful in enhancing perception and facilitating visual search. In contrast to SA, however, FBA has been studied almost exclusively in terms of its endogenous deployment. Endogenous FBA can be allocated to a wide range of visual features, such as a particular color, an orientation, or a motion direction (for review, see Carrasco, 2011; Serences \& Kastner, 2014), and simultaneously to multiple feature values (Liu, Becker, \& Jigo, 2013; Liu \& Jigo, 2017). It spreads across space, even to irrelevant or unattended locations (Andersen, Hillyard, \& Müller, 2013; Liu \& Mance, 2011; Rossi \& Paradiso, 1995; Saenz, Buracas, \& Boynton, 2002; Serences \& Boynton, 2007; Störmer \& Alvarez, 2014; Treue \& Martínez-Trujillo, 1999; White \& Carrasco, 2011). Endogenous FBA is deployed more slowly than endogenous SA, taking around $500 \mathrm{~ms}$ rather than $300 \mathrm{~ms}$ (Carrasco, 2011; Liu et al., 2007b; Serences \& Kastner, 2014), and its neural bases are well characterized (Müller, 2014; Treue, 2014; Treue \& Martínez-Trujillo, 2007). Moreover, endogenous FBA can interact with endogenous SA (e.g., Andersen, Fuchs, \& Müller, 2011; Hayden \& Gallant, 2009; Patzwahl \& Treue, 2009; Treue \& Martínez-Trujillo, 1999; White, Rolfs, \& Carrasco, 2015).

The selectivity afforded by FBA and its interaction with SA is particularly useful in visual search - that is, when searching for a target stimulus that has known features but whose location amidst distracters with different features is unknown. Visual search is a central paradigm, which, starting with the seminal research of Anne Treisman (e.g., Treisman, 1982; Treisman, 1988; Treisman \& Gelade, 1980), has been used to study our ability to detect, discriminate or localize a target among distracters (for reviews, see Eckstein, 2011; Eimer, 2014; Nakayama \& Martini, 2010). In a "feature search" (e.g., an observer is instructed to detect a red tilted target amongst red vertical distractors), it is useful for the observer to grant priority in processing relevant features and/or to enhance their representation. In a "conjunction search," an observer may be instructed to detect a red tilted target, or to discriminate its tilt (left vs. right), amidst blue tilted and red vertical distracters. In this case, it would be useful for the observer to prioritize the processing of red and/or tilted items. Indeed, some authors have proposed that an early stage of the search process is to select the subset of stimuli that contain at least one of the target's features (e.g., Egeth, Virzi, \& Garbart, 1984; McElree \& Carrasco, 1999; Wolfe \& Horowitz, 2004). Support for this proposal comes from studies in which cueing relevant features (either size or color) aid performance in visual search tasks, under some conditions, by prioritizing processing of those stimuli and guiding SA to them before others (e.g., Moore \& Egeth, 1998; Shih \& Sperling, 1996). Also relevant to the role of FBA in visual search is the finding that the effect of feature guidance increases when a selection bias can build over successive trials because the target feature remains the same from trial to trial (e.g., Carrasco, Ponte, Rechea, \& Sampedro, 1998; Müller, Heller, \& Ziegler, 1995; Wolfe, Butcher, Lee, \& Hyle, 2003; Wolfe \& Horowitz, 2004).

In contrast to endogenous FBA, very little is known about a potential exogenous form of FBA. Two relatively recent studies explicitly investigated the potential for exogenous FBA to influence perception, using a similar approach (Lin, HubertWallander, Murray, \& Boynton, 2011; Qian \& Liu, 2015). A comprehensive characterization of exogenous FBA would be useful to understand its possible role in visual search and other functions. For example, in a similar fashion to the studies in which SA has been explicitly manipulated in the investigation of visual search (Cameron, Tai, Eckstein, \& Carrasco, 2004; Carrasco, Giordano, \& McElree, 2004a, 2006; Carrasco \& McElree, 2001; Giordano, McElree, \& Carrasco, 2009; Grubb, Behrmann, Egan, Minshew, Carrasco, et al., 2013a; Grubb, Behrmann, Egan, Minshew, Heeger, et al., 2013b; Yeshurun \& Carrasco, 1998), we could investigate how explicitly manipulating exogenous FBA would impact performance in a variety of detection, discrimination and localization tasks.

Moreover, given our recent findings regarding the influence of covert attention on perceptual learning, we viewed exogenous FBA as a potential tool for affecting perceptual learning. Specifically, we have found that other forms of covert attention - exogenous spatial attention (Donovan, Szpiro, \& Carrasco, 2015; Szpiro \& Carrasco, 2015); endogenous spatial attention (Donovan \& Carrasco, 2018); and endogenous FBA (Cavanaugh, Barbot, Carrasco, \& Huxlin, 2019)can potentiate perceptual learning and reduce the retinal specificity of learning. Exogenous forms of attention are especially promising for perceptual learning in clinical populations, as they require no additional effort from the observer. Before investigating how exogenous FBA affects visual search and perceptual learning, we aimed to characterize its temporal dynamics to ensure we could optimize its possible effects in these domains.

In the first study of exogenous FBA (Lin et al., 2011), participants detected the presence (Experiment 1) or discriminated the orientation (Experiment 3 ) of an oval in an array of uniquely colored circles appearing $200 \mathrm{~ms}$ after an uninformative precue was briefly presented. In most studies that manipulate endogenous FBA, a precue has been used as well (e.g., Adamian, Slaustaite, \& Andersen, 2019; Fang, Becker, \& Liu, 2019; Herrmann, Heeger, \& Carrasco, 2012; Ling, Liu, \& Carrasco, 2009; Liu et al., 2007b; McMains, Fehd, 
Emmanouil, \& Kastner, 2007; Serences \& Boynton, 2007; Störmer \& Alvarez, 2014; White \& Carrasco, 2011; White et al., 2015). Exogenous SA and exogenous FBA were probed by independently varying the precue's spatial location and color relative to the target ("valid" meaning matching that of the target, "invalid" meaning differing from the target). Attentional effects were measured by reaction time differences between "valid" and "invalid" trials in both detection (Experiments 1) and discrimination (Experiment 3) tasks. They found that in both Experiments, RT was faster for feature-valid trials than for feature-invalid trials.

In the second study (Qian \& Liu, 2015), a similar design to Lin et al. (2011) was used, except that the precue was presented very briefly $(\sim 20 \mathrm{~ms})$ between a forward and a backward mask, with the intent of making the feature content of the precue imperceptible to the observer (Qian \& Liu, 2015). Two interstimulus intervals (ISIs; time between precue offset and target onset) were used in two separate experiments (500 ms in Experiment 1; $200 \mathrm{~ms}$ in Experiment 2). Interestingly, their results showed that participants responded faster for feature-valid trials, but this FBA effect appeared only for ISI as long as $500 \mathrm{~ms}$. This was the case even when the precue was presented longer $(100 \mathrm{~ms})$ and rendered conscious (Experiment 3). To try to reconcile their finding with those of Lin et al. (2011), the authors suggested that the masks may have delayed the visual processing of the precue, hence delaying the exogenous FBA effect such that it was not found with the $200 \mathrm{~ms}$ ISI used in Lin et al. (2011).

These two studies (Lin et al., 2011; Qian \& Liu, 2015) suggested that FBA can be deployed exogenously, even without conscious awareness (Qian \& Liu, 2015). It is crucial to note that the exogenous FBA effects on performance were found for very different ISIs. Whereas Lin et al. (2011) reported that exogenous FBA was present as early as $200 \mathrm{~ms}$ after cue offset, Qian and Liu (2015) reported that it takes more than $200 \mathrm{~ms}$ for exogenous FBA to take effect. Thus, it remains unclear whether these FBA effects are transient and what is the optimal time between the cue and the target for exogenous FBA to actually influence performance.

Additionally, both studies used reaction time (RT) as their main measure of performance. RT is limited in its ability to address the temporal dynamics of perceptual processes because faster reaction times could arise from any combination of improved discriminability, faster speed of processing, criterion shifts, or motor components (Carrasco \& McElree, 2001; McElree \& Dosher, 1989; Reed, 1973). Moreover, in both studies the stimulus display remained on the screen until the participant's response (several hundred milliseconds). Thus, there was a rather long range of time at which the cue could have affected perception of the target, given the stimulus display was long enough for a transient mechanism like exogenous SA to peak and decline to baseline. Whether the FBA effects were transient could not be established because observers were able to search the display until the target was found, and even saccade to several distractor and target locations. These factors further obscure the temporal dynamics of the reported exogenous FBA effects. Given the limitations of the two investigations of exogenous FBA, its potential effects on perception and its temporal dynamics are unclear.

Perhaps due to this long-standing gap in knowledge, some had asserted that there is no distinction between endogenous FBA and bottom-up priming effects (Awh, Belopolsky, \& Theeuwes, 2012; Theeuwes, 2013; but see Belopolsky \& Awh, 2016; van Es, Theeuwes, \& Knapen, 2018). Note that this view conflicts with several findings that can only be accounted for by top-down orienting to particular feature values (e.g., Belopolsky \& Awh, 2016; Herrmann et al., 2012; Liu, Hospadaruk, Zhu, \& Gardner, 2011; Liu, Larsson, \& Carrasco, 2007a; Liu, Slotnick, Serences, \& Yantis, 2003b; Liu et al., 2007b; Serences \& Yantis, 2007; White \& Carrasco, 2011; White, Rolfs, \& Carrasco, 2013; White et al., 2015). In any case, in order to have a comprehensive understanding of the factors that limit and alter perception and performance, it is crucial to verify and characterize the effects of exogenous FBA, as well as to distinguish them from those of endogenous FBA.

The goal of the current study is to further characterize exogenous FBA and to investigate its time course. We adapted the experimental procedure used by Lin et al. (2011), also similar to that by Qian \& Liu (2015). A strength of this design is that the feature dimension of the precue - color - is orthogonal to the task-relevant featureorientation. FBA is known to affect the processing of taskirrelevant features that are associated with the attended feature (Katzner, Busse, \& Treue, 2009; Schoenfeld et al., 2007; Sohn, Chong, Papathomas, \& Vidnyánszky, 2005; White \& Carrasco, 2011), so in principle, directing attention to a stimulus via one dimension can enhance the processing of another feature of that stimulus. In the first experiment, to have a baseline for the subsequent experiments we attempted to replicate the effects of Lin et al. (2011) by using nearly identical parameters (most closely matched to their Experiment 3), and focused on RT as the primary performance measure (as in the two previous studies on exogenous FBA). In later Experiments, we used three ISIs $-100 \mathrm{~ms}, 200 \mathrm{~ms}$, and $300 \mathrm{~ms}$; or $100 \mathrm{~ms}, 300 \mathrm{~ms}$, and $500 \mathrm{~ms}$ - and assessed both accuracy and RT to assess the effects of exogenous FBA on visual performance. Additionally, in Experiments 2-4, we had a brief display (60 ms) of targets and distractors and used eye tracking to ensure fixation throughout each trial. These parameters enabled us to probe specific time windows and investigate exogenous FBA's time course, as well as its possible transient effect on perception. In all experiments, the feature cue was $50 \%$ valid to maximize the number of trials for each cue type (valid vs. invalid). 


\section{Experiment 1}

\section{Method}

\section{Participants}

Fourteen participants (11 female) with normal or corrected-tonormal vision were recruited to participate in the experiment. We conducted a power analysis using G*Power (Faul, Erdfelder, Lang, \& Buchner, 2007; power $=0.80$, partial eta squared $=0.42$ ) and found that this sample size is consistent with the effect size (partial eta squared) reported by Lin et al. (2011). All observers were naïve to the purposes of the study, had normal color vision, and had normal or corrected-tonormal vision (glasses or contacts). The NYU Institutional Review Board approved this study.

\section{Experimental design}

We followed the designs of Lin et al.'s (2011) Experiment 3. We used a 2 (feature validity: valid or invalid) $\times 2$ (spatial validity: valid or invalid) within-subjects design, with an ISI of $200 \mathrm{~ms}$ between cue offset and stimulus onset.

\section{Apparatus}

Stimuli were generated using PsychToolbox (Brainard, 1997; Pelli, 1997) in MATLAB (The MathWorks, Natick, MA) and were displayed on a 21-in. CRT monitor $(1,280 \times 960$ at 85 $\mathrm{Hz})$. Eye position was monitored using an infrared eye tracker (EyeLink 1000 CL, SR Research, Kanata, Ontario, Canada).

\section{Stimuli}

Six filled-in disks (diameter 1.8 degrees of visual angle [dva]) of six different solid colors were presented on a black background. The discs would appear at six out of eight possible locations, representing both "cardinal" (left, right, up, down) and "intercardinal" (upper left, upper right, lower left, lower right) isoeccentric locations, $5.03 \mathrm{dva}$ from fixation. One of the discs was an ellipse, and the rest were circles. The ellipse was compressed on one axis, either vertically or horizontally, by a magnitude determined by a threshold estimate from a QUEST procedure (see the Procedure section, below). The 60 -ms precue, a square $(0.92 \times 0.92 \mathrm{dva})$ filled with a solid color, matching the color of one of the discs, was presented $200 \mathrm{~ms}$ before stimuli display at one of the possible target or distractor locations. Target color and distractors were selected without replacement from the following pool of colors (RGB color vector values: red $[255,0,0]$, green $[0,255,0]$, blue $[0$, $0,255]$, yellow [255, 128, 0], cyan [0,128, 255], and purple/ magenta $[255,0,128])$.

\section{Procedure}

Figure 1 shows the trial sequence. Participants were seated in a dimly lit room, with their heads placed on the chin rest $57 \mathrm{~cm}$ from the screen. At the beginning of each session, participants were given the following prompt as the only instructions for the task (besides clarification questions answered by the experimenter):

"At the beginning of every trial you will be presented with a colored square, then five circles and one oval. Your task today is to locate the oval among circles as quickly as possible and identify the orientation of the oval. On $50 \%$ of trials, the oval will be vertical and you should press the ' $V$ ' key. In the other $50 \%$ of trials, the oval will be horizontal and you should press the ' $\mathrm{H}$ ' key. In every trial, you will see a square cue presented before the oval circles. Sometimes this cue may be the same color as the target oval. Sometimes this cue will be at the same location as the target oval, and sometimes the cue will have nothing in common with the target oval. The full experiment is 12 blocks of 90 trials. You will get breaks between each block. Please respond as quickly as possible, but maintain at least $88 \%$ accuracy! Good luck!

Eye movements were monitored, but fixation was not mandatory after $200 \mathrm{~ms}$ of initial fixation. The trial would begin with the presentation of a white fixation circle $(1 \times 1 \mathrm{dva})$ at the center of the screen. Before stimulus onset, the square precue (see the Stimuli section) would appear at either the target location (spatial valid) or one of the distractor locations (spatial invalid), and would either match the color of the target (feature valid) or one of the distractors (feature invalid). Thus, there were four precue types, all equally likely: (1) spatial valid and feature valid, (2) spatial valid and feature invalid, (3) spatial invalid and feature valid, and (4) spatial invalid and feature invalid. The precue was followed by a $200-\mathrm{ms}$ ISI, and then the target display was presented (see the Stimuli section). Participants had 2,000 ms to report whether the target oval was horizontally or vertically oriented by pressing one of two keys (see Fig. 1 for a schematic illustration of trial display). Auditory feedback was provided after each response. Trials of different spatial and feature validities were randomly intermixed within a block. Each participant completed two sessions of 12 blocks with 90 trials each, resulting in 2,160 trials in total, split evenly between two sessions on separate days. This is 4 times as many trials as in Lin et al. (2015).

To ensure that the task difficulty was at an intermediate level, participants first completed two blocks of a modified QUEST procedure (Watson \& Pelli, 1983) to determine the compression magnitude of the target oval to reach 


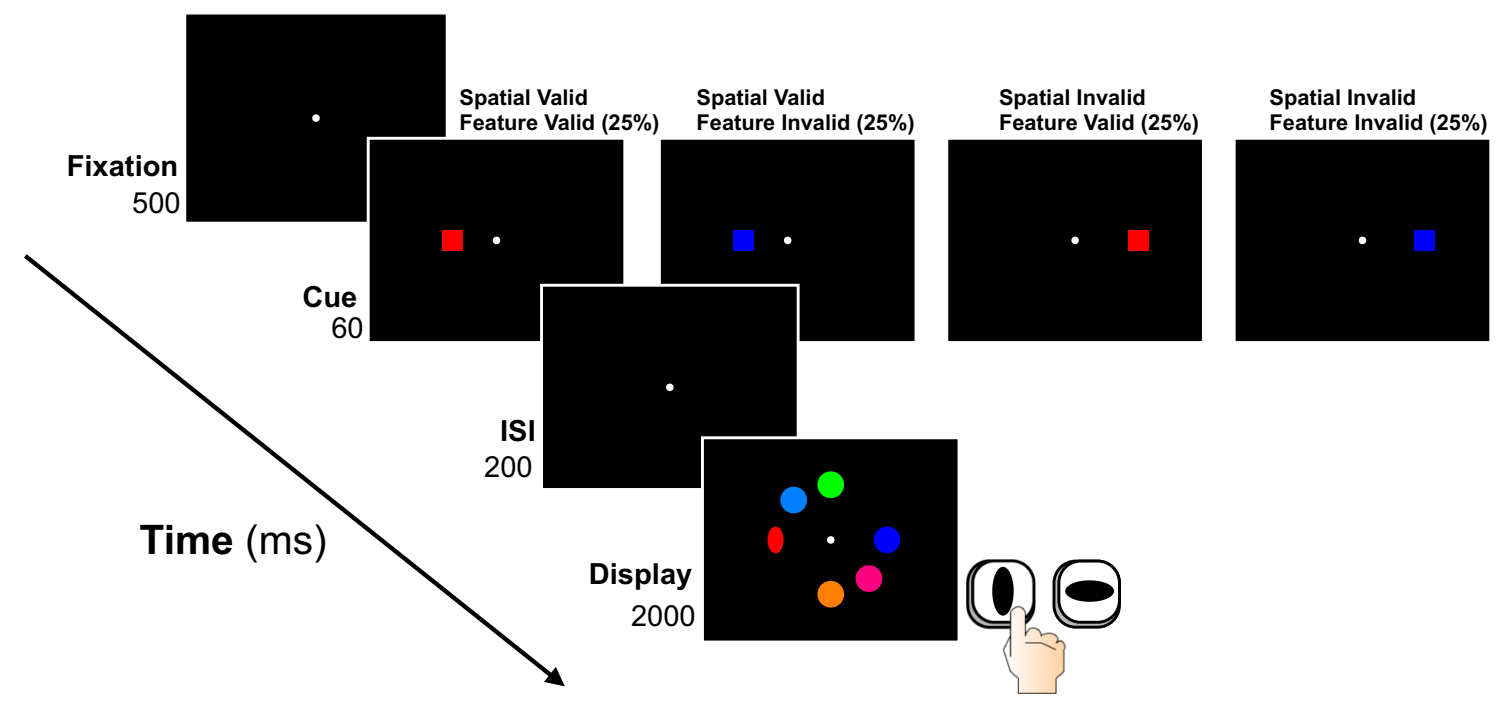

Fig. 1 Trial sequence for Experiment 1

approximately $90 \%$ accuracy. Our primary measure of performance was RT, and we ensured accuracy fell between $88 \%$ and $95 \%$ throughout all blocks. We did this because accuracy above $95 \%$ is too close to ceiling performance and indicates that participants could be responding more quickly, and below $88 \%$ accuracy RT trades off heavily with small changes in accuracy and vice versa (Pachella, 1973). To ensure that task difficulty was maintained during the main experiment, participants were instructed to achieve approximately $90 \%$ accuracy, and the experimental sequence would pause if participant's average accuracy in the previous two blocks fell below $88 \%$ or above $95 \%$. Participants were instructed to respond as fast as possible (if accuracy was too high) or be more accurate and less fast (if accuracy was too low). If accuracy remained outside of the range of $88 \%-95 \%$, the amount of compression would be adjusted manually by $1 \%-3 \%$-increasing compression if accuracy was too high and decreasing compression if accuracy was too low.

Notably, in a preliminary pilot, we attempted to use the same compression ratio for all observers as in Lin et al. (2011), which was $95 \%$ of the uncompressed axis for all observers. This resulted in accuracy that was too low (mean = $74.80 \%$, standard error $=4.67 \%, n=6$ ) so RT results were not meaningful. Based on this pilot, we decided to add the initial QUEST staircase, and to manually adjust difficulty between blocks if needed, as described above.

\section{Results}

RT and accuracy are shown in Fig. 2. All observers had an overall accuracy between $88 \%$ and $95 \%$ correct (mean = $93.42 \%, S E M=0.73 \%$ ). This allowed us to conduct a twoway (Feature Validity $\times$ Spatial Validity) analysis of variance (ANOVA) on RT (median of the correct trials within each condition). There was no interaction between feature validity and spatial validity, $F(1,13)<1$, no main effects of spatial validity, $F(1,13)=1.664, p=.220$, or feature validity, $F(1,13)$ $=2.035, p=.177$. Despite the lack of a significant two-way interaction, for exploratory reasons, and because Lin et al. (2011)'s data in Fig. 2 suggests that the feature validity effect was driven by the invalid conditions, we analyzed the feature validity effect in each of the two spatial validity conditions. we found no significant effect of feature validity either in the spatial-valid, $t(13)=1.667, p=.119$, or in the spatial-invalid,

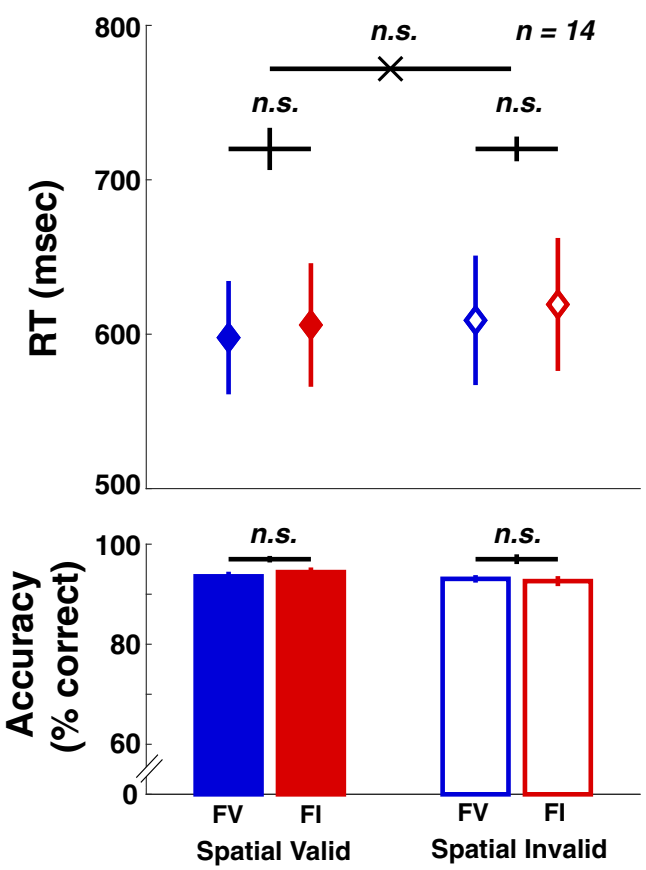

Fig. 2 Median RT (correct trials) and mea1n accuracy from Experiment 1. Blue bars and points represent feature-valid trials; red represents feature-invalid trials. Solid bars and points indicate spatial-valid (SV) trials; hollow bars and points indicate spatial-invalid (SI) trials. Error bars indicate standard error of the mean. (Color figure online) 
$t(13)=0.902, p=.384$, conditions. We repeated the analysis using geometric means and found the same pattern of results.

We also conducted a Bayesian model selection analysis (Masson, 2011). To do so, we transformed the sum of squared errors obtained from our ANOVAs to arrive at an estimated Bayes factor as well as Bayesian information criterion probabilities $\left(p\right.$ BIC) for the alternative $\left(\mathrm{H}_{1}\right)$ hypotheses given Data Set $\mathrm{D}$. Here, we report the probability of the alternative hypotheses - $p \mathrm{BIC}\left(\mathrm{H}_{1} \mid \mathrm{Data}\right)$ - which shows positive evidence for the alternative hypothesis above 0.75 , and positive evidence for the null hypothesis below 0.25 . In between those values, the evidence is inconclusive (Masson, 2011; Raftery, 1995). This analysis indicated inconclusive results for the feature-validity main effect $-p \mathrm{BIC}\left(\mathrm{H}_{1} \mid \mathrm{Data}\right)=0.4245$ - and the spatial-validity main effect $-p \mathrm{BIC}\left(\mathrm{H}_{1} \mid\right.$ Data $)=0.3822$. The Feature Validity $\times$ Spatial Validity interaction showed positive evidence for the null hypothesis - that is, no effect $-p \mathrm{BIC}\left(\mathrm{H}_{1} \mid\right.$ Data $)=0.2132$.

\section{Discussion}

Our first goal was to replicate the original finding of exogenous FBA improving RT, as reported in Lin et al. (2011). We used the same task, ISI length, stimulus features (size, color, location), number of distractors, and very similar instructions (based on instructions shared with us by one of the authors, G. Boynton). We found neither an effect of feature validity nor of spatial validity, and Bayesian model selection analysis indicated these effects were inconclusive. The lack of a spatial-validity effect is likely because exogenous SA peaks before $150 \mathrm{~ms}$ after cue onset (Cheal \& Lyon, 1991a, 1991b; Müller \& Rabbitt, 1989; Nakayama \& Mackeben, 1989), and the SOA in this experiment was $>250 \mathrm{~ms}$. In subsequent experiments, we altered the experimental design to address several plausible concerns as to why a feature-validity effect eluded this initial attempt.

\section{Experiment 2}

Although Experiment 1 did not reveal a significant effect of an exogenous FBA cue despite similarities to the previous studies, there were several aspects of the procedure that we believed might have prevented a robust effect. First, participants were not required to fixate throughout the trial, and the stimuli were displayed for 2 seconds. This means that participants could have made saccades to multiple locations before making a response, thus obscuring any exogenous influence of the precue on the processing of the target, as overt eye movements may assist in search and performance. Second, the long presentation of the target display is problematic, even with fixation contingency, because the temporal dynamics of exogenous FBA have not been characterized. Thus, such a long stimulus display does allow determination of when an exogenous FBA precue must appear before stimulus display in order to influence performance. Third, RT is limited as a behavioral measure, as it could reflect changes in speed of processing, discriminability, criterion, or motor responsiveness (Carrasco \& McElree, 2001; McElree \& Dosher, 1989; Reed, 1973). Even though we ensured that RT was not confounded with accuracy, a lack of an effect in RT does not rule out that the FBA cue could influence perception. Finally, subjectively, we noticed that with a black background, there was a luminance "halo" around the colored discs, as the glass of the screen diffused some of the light emitted around the discs.

In this experiment, we altered the experimental procedure to circumvent these methodological concerns. Specifically, we presented the stimulus for a brief amount of time to prevent eye movements, ensured fixation throughout each trial using online eye tracking, focused on accuracy rather than RT as the primary behavioral measure, and used a gray background to avoid blurring the edges of the stimulus. Additionally, to investigate how the time between the onset of the cue and stimulus may influence the effect of the precue on performance, we used three different ISI conditions: the original ISI (200 $\mathrm{ms})$, one shorter (100 ms), and one longer (300 ms).

\section{Method}

\section{Participants}

Fourteen participants (eight female) were recruited to participate in the experiment. All observers were naïve to the purposes of the study, had normal color vision, and had normal or corrected-to-normal vision (glasses or contacts). The NYU Institutional Review Board approved this study.

\section{Apparatus}

The apparatus as the same as in Experiment 1.

\section{Experimental design}

We used a very similar procedure as in Experiment 1, with the addition that three different ISI conditions were tested on separate blocks. As a result, this Experiment had a 2 (feature validity: valid or invalid) $\times 2$ (spatial validity: valid or invalid) $\times 3$ (cue-target ISIs: $100 \mathrm{~ms}, 200 \mathrm{~ms}, 300 \mathrm{~ms}$ ) within-subjects design. The 100-ms ISI represents an ISI time at which exogenous SA is near peak (Cheal \& Lyon, 1991a, 1991b; Müller \& Rabbitt, 1989; Nakayama \& Mackeben, 1989) and therefore could theoretically result in exogenous FBA effects if the two mechanisms share similar time courses. The $300-\mathrm{ms}$ ISI is too short for FBA to be deployed endogenously in response to the cue (Liu et al., 2007b), and too long for exogenous SA to exert an effect (Nakayama \& Mackeben, 1989). The 200-ms ISI was chosen because it is the short and long ISIs. 


\section{Stimuli}

The stimuli were the same as those in Experiment 1, except that the background was mean-luminance gray instead of black, and the fixation cross was black instead of white.

\section{Procedure}

Figure 3 shows the trial sequence. The procedure was identical to Experiment 1, except that (i) Participants were required to fixate on a central black fixation circle $(1 \times 1 \mathrm{dva})$ for the duration of each trial, except for the response window; (ii) variable ISIs were used between the precue and target displays (see Experimental Design section); (iii) the brief target display of $60 \mathrm{~ms}$; (iv) responses could be provided any time after stimulus onset, with a 2,000 ms time limit. Each participant completed four blocks of 96 trials at each ISI condition, resulting in 1,152 trials in total. Accuracy was used as the primary behavioral measures, and RT was also recorded as a secondary dependent variable.

To ensure that the task difficulty was set to an intermediate level, participants completed one block of 100 trials of a threedown one-up staircase procedure conducted before the experiment to determine the compression magnitude of the target oval for each subject to reach approximately $83 \%$ accuracy (García-Pérez, 1998). We used a 3-1 up staircase in this and supsequent Experiments instead of the unconventional modified QUEST procedure from Experiment 1 that was used to target a compression ratio that resulted in $90 \%$ accuracy. The more conventional staircase titrates for intermediate accuracy, which is ideal when accuracy is the primary behavioral measure, as it is in Experiments 1, 2, and 3.

\section{Results}

Accuracy and RT are shown in Fig. 4a, separated by feature validity, ISI, and spatial validity conditions. We conducted a three-way (Feature Validity $\times$ Spatial Validity $\times$ ISI) ANOVA on accuracy. There was no three-way interaction, Greenhouse-Geisser correction, $F(1,19.693)<1$; no twoway interaction of feature validity and spatial validity, Greenhouse-Geisser correction, $F(1,13)<1$; no main effect of ISI, Greenhouse-Geisser correction, $F(1,25.685)<1$; and no main effect of feature validity, $F(1,13)=2.981, p>.10$. There was a two-way interaction between spatial validity and ISI, Greenhouse-Geisser correction, $F(1,25.513)=7.086, p<$ .01 , and there was a main effect of spatial validity, $F(1,13)=$ $6.194, p<.02,87.03 \%$ for valid and $81.98 \%$ for invalid. The interaction between ISI and spatial validity was driven by the higher accuracy for spatial valid than spatial invalid in the 100 -ms ISI, $t(1,13)=3.937, p=.02$, although accuracy for spatial valid was always greater than for spatial invalid across all ISIs (see Fig. 4b). We analyzed RT and verified that there was no speed-accuracy tradeoff.

We conducted the same Bayesian analysis as in Experiment 1. The following effects and interactions support the null hypothesis: ISI $\times$ Space $\times$ Feature interaction $p \mathrm{BIC}\left(\mathrm{H}_{1} \mid\right.$ Data $)=$ 0.1427 ; Feature $\times$ ISI interaction $p \mathrm{BIC}\left(\mathrm{H}_{1} \mid\right.$ Data $)=0.0743$; ISI main effect $p$ BIC $\left(\mathrm{H}_{1} \mid\right.$ Data $)=$

0.0738 . The following effects were inconclusive: Feature $\times$ Space interaction $p \mathrm{BIC}\left(\mathrm{H}_{1} \mid\right.$ Data $)=0.2812$; ISI $\times$ Space interaction $p \mathrm{BIC}(\mathrm{H} 1 \mid \mathrm{Data})=0.6125$; and feature main effect $p \mathrm{BIC}\left(\mathrm{H}_{1} \mid\right.$ Data $)=0.5324$. The following show positive evidence for the alternative hypothesis: space main effect $p \mathrm{BIC}\left(\mathrm{H}_{1} \mid\right.$ Data $)=0.9814$.

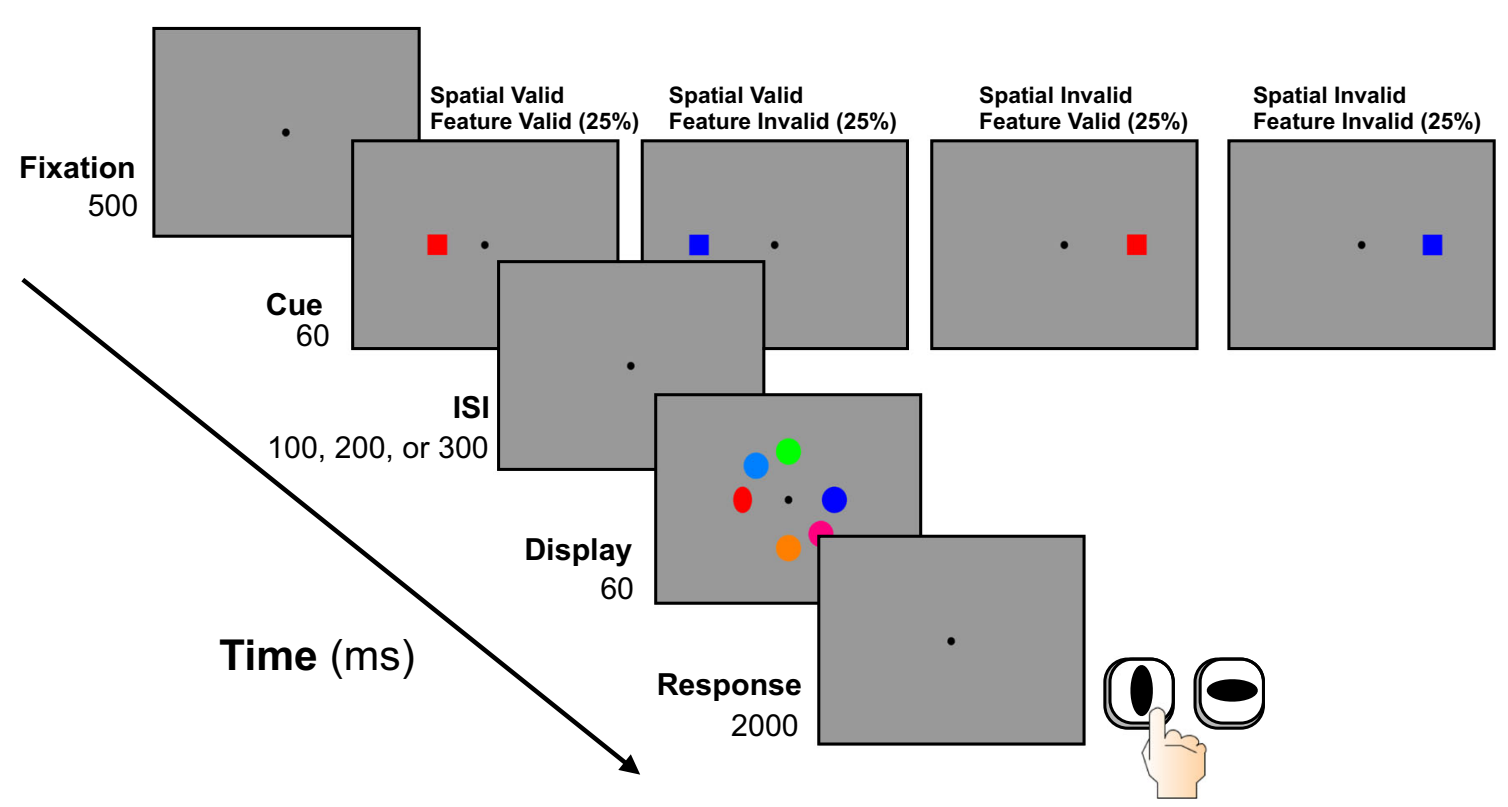

Fig. 3 Trial sequence for Experiment 2 


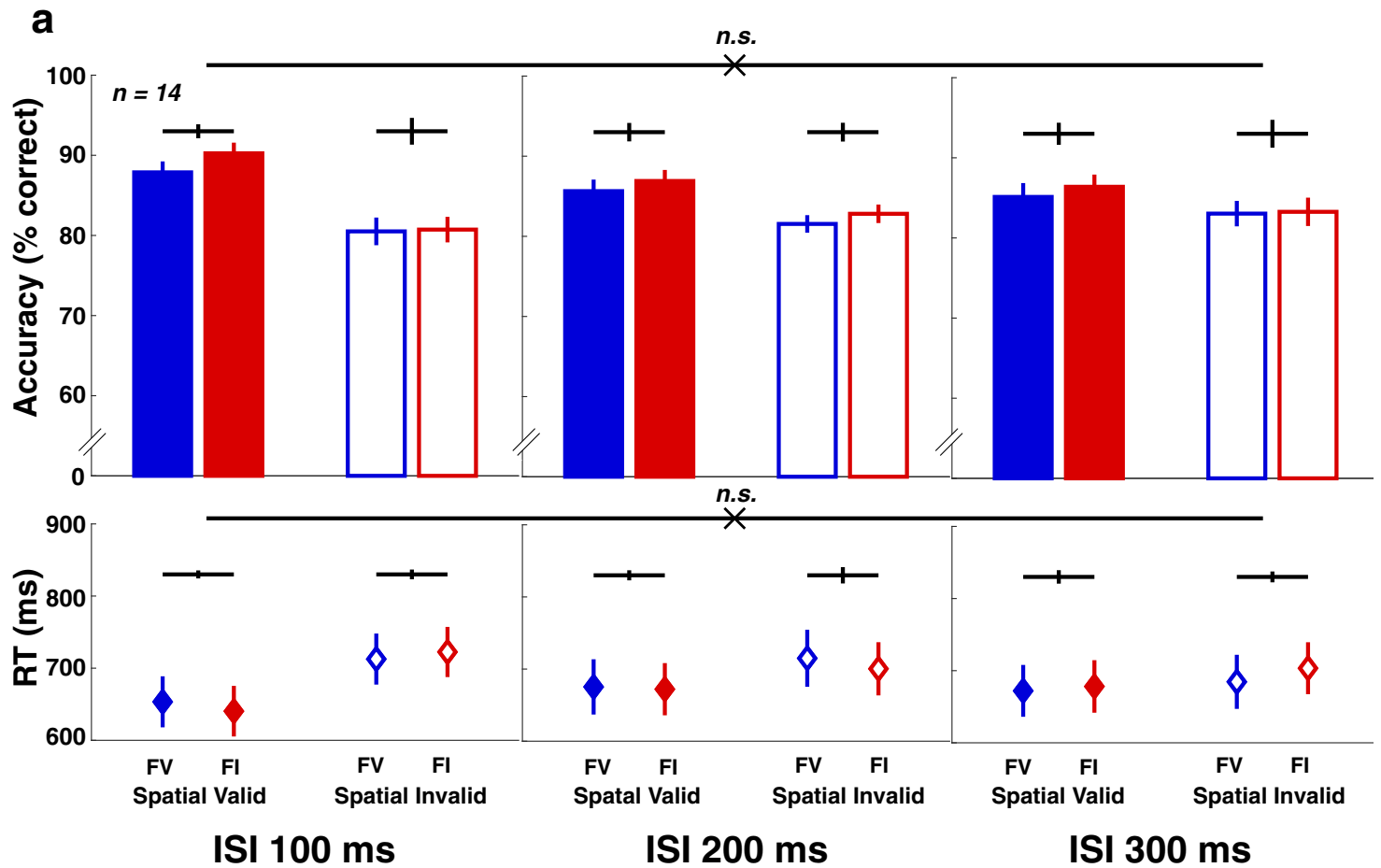

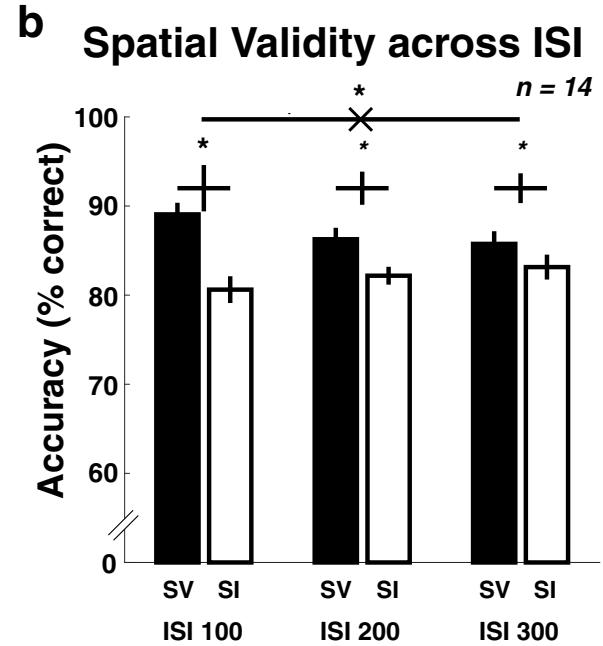

Fig. 4 Results from mean accuracy and median RT from Experiment 2. Top panel (a) shows all conditions separately, while bottom panel shows data divided by the significant two-way interactions. a Mean accuracy and median RT across ISI, spatial validity, and feature validity. Blue bars and points represent feature-valid trials, while red represents featureinvalid trials. Solid bars and points indicate spatial-valid (SV) trials, while hollow bars and points indicate spatial-invalid (SI) trials. Separate panels

\section{Discussion}

In this experiment, we again found no significant effect of feature validity. The significant main effect of spatial validity and the significant interaction of ISI and spatial validity are consistent with previous research (e.g., Carrasco, Ling, show data for each ISI condition. Error bars indicate standard error of the mean. Black error bars indicate the standard error of the mean of the difference between the adjacent conditions (FV - FI). b Accuracy for each ISI and spatial validity condition, collapsed across feature validity. Solid bars show spatial-valid data, and hollow bars show spatial-invalid conditions. All error bars indicate standard error of the mean. (Color figure online) 
using this design, was sensitive to selective attention despite no effect of feature validity in this experiment.

\section{Experiment 3}

Endogenous SA and endogenous FBA can interact, and whether they do so in an additive or a superadditive fashion depends on the level of stimulus competition (White et al., 2015). Although it has not been established whether exogenous SA and exogenous FBA interact, the effects of exogenous FBA reported by Lin et al. (2011) with peripheral cues at either target or distractor locations (Experiments 1 and 3), but not with central, spatially neutral cues (Experiment 2), suggest they may do so. Thus, we considered whether the inconsistent main effect of FBA, as well as the consistent lack of a Feature Validity $\times$ ISI interaction in the previous experiment, could have been due to a possible local interaction between exogenous FBA and exogenous SA. For example, if exogenous SA and exogenous FBA had different time courses, at a particular ISI the effect of one on performance could have obscured the effect of the other. Given that endogenous FBA spreads across space even to irrelevant and unattended locations (Andersen et al., 2013; Liu \& Mance, 2011; Rossi \& Paradiso, 1995; Saenz et al., 2002; Serences \& Boynton, 2007; Störmer \& Alvarez, 2014; Treue \& MartínezTrujillo, 1999; White \& Carrasco, 2011), we moved the location of the precue from the periphery to fixation, and thus its validity was only in terms of feature (feature valid or feature invalid), as has been done in numerous studies of endogenous FBA (e.g., Jigo, Gong, \& Liu, 2018; White et al., 2015).

\section{Method}

\section{Participants}

Fifteen participants ( 10 female) were recruited to participate in the experiment. All observers were naïve to the purposes of the study, had normal color vision, and had normal or corrected-to-normal vision (glasses or contacts). The NYU Institutional Review Board approved this study.

\section{Apparatus}

The apparatus was the same as in Experiments 1 and 2.

\section{Stimuli and experimental design}

Figure 5 shows the trial sequence. The stimuli were identical to Experiment 2. We used a procedure similar to the one in Experiment 2, except that precues were always presented over the center of the fixation cross and thus were neutral with regard to its spatial location (i.e., not matching the location of the target or any distractors). As a result, this Experiment had a 2 (feature validity: valid or invalid) $\times 3$ (cue-target ISIs: $100 \mathrm{~ms}, 200 \mathrm{~ms}, 300 \mathrm{~ms}$ ) within-subjects design. Again, ISI was consistent within each block.

\section{Procedure}

The procedure was the same as in Experiment 2, except that the precue appeared at fixation instead of peripherally. The

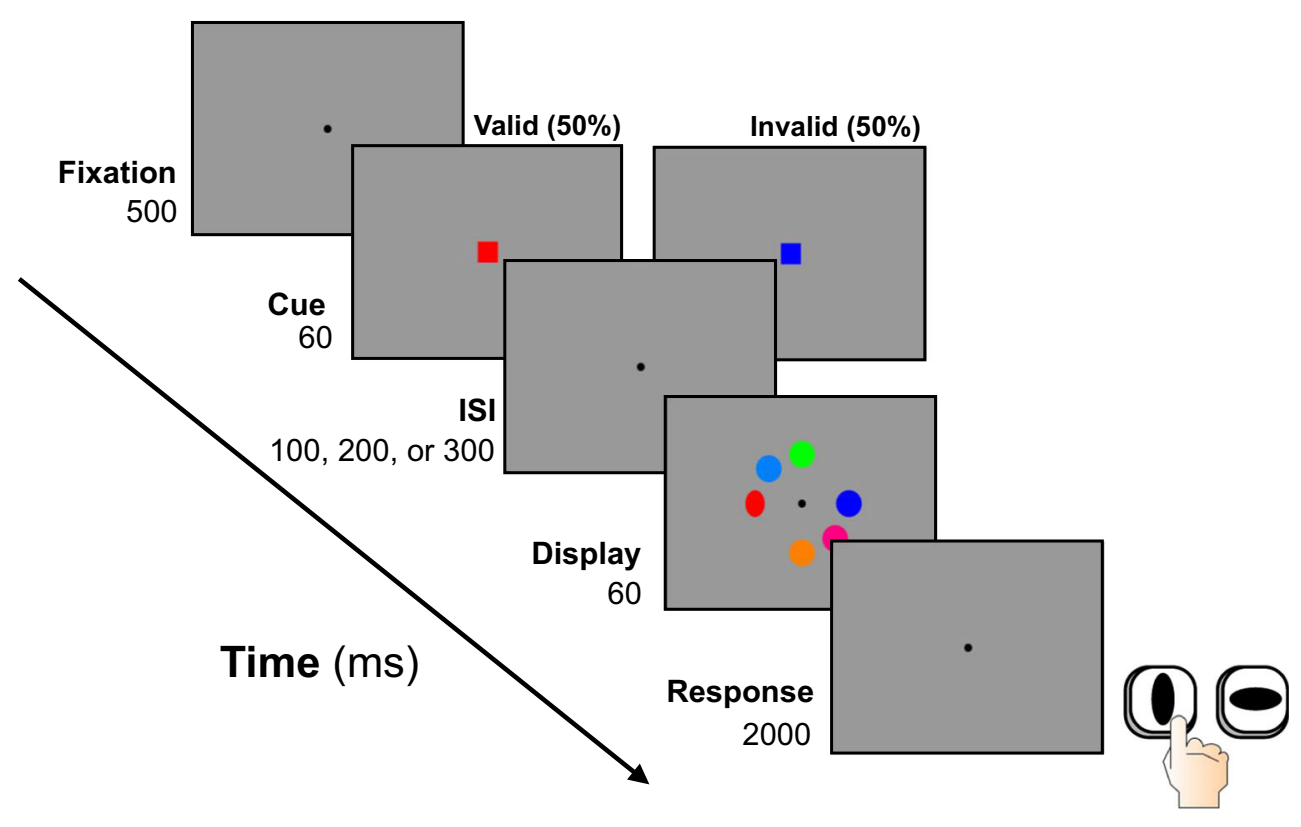

Fig. 5 Trial sequence for Experiment 3 
feature validity of the precue was $50 \%$, and trials of different feature validities were interleaved within a block. Each participant completed four blocks of 96 trials at each ISI condition, resulting in 1,152 trials in total. Accuracy was used as the primary behavioral measure, and RT was recorded as a secondary measurement.

\section{Results}

Accuracy and RT are shown in Fig. 6, separated by ISI and feature validity conditions. We conducted a two-way (Feature Validity $\times$ ISI) analysis of variance (ANOVA) on accuracy. There was no two-way interaction, Greenhouse-Geisser correction, $F(1,26.044)<1$. There was neither a main effect of ISI, Greenhouse-Geisser correction, $F(1,27.043)<1$, nor a main effect of feature validity, Greenhouse-Geisser correction, $F(1,14)=2.173, p=.163$. We analyzed RT and verified that there was no speed-accuracy trade-off.

We conducted the same Bayesian analysis as in the previous experiments. This analysis indicated inconclusive results for the feature validity main effect $-p \mathrm{BIC}(\mathrm{H} 1 \mid$ Data $)=$ 0.4532 - and the ISI main effect $-p$ BIC $($ H1 $\mid$ Data $)=0.4510$. The interaction of feature validity and ISI showed positive evidence for the null hypothesis - that is, no effect$p \mathrm{BIC}(\mathrm{H} 1 \mid$ Data $)=0.2077$.
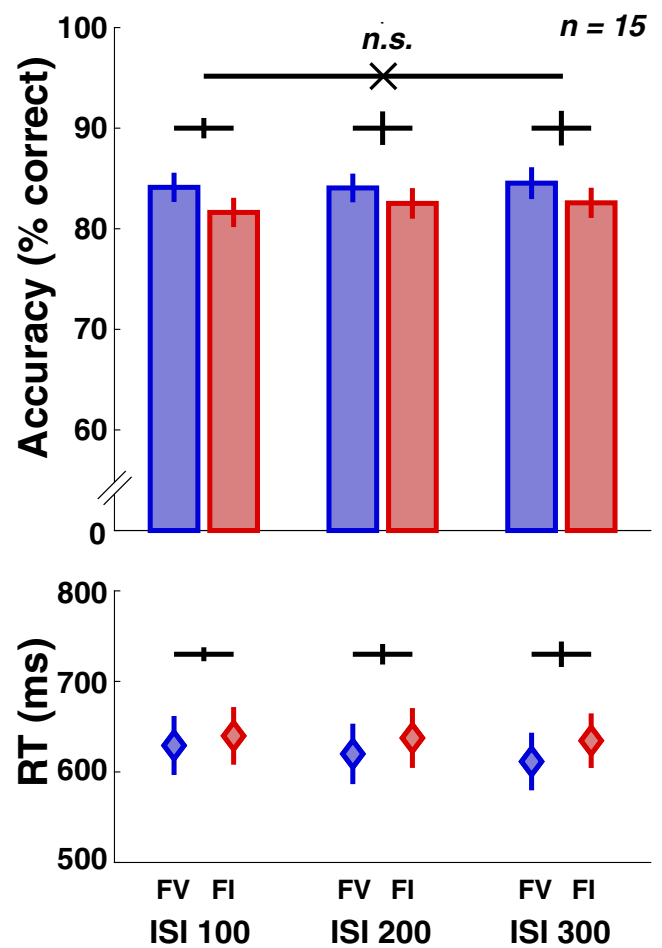

Fig. 6 Mean accuracy and median RT from Experiment 3. Blue bars and points represent feature-valid trials; red represents feature-invalid trials. Blue and red pairs show data for each ISI condition. Lighter colors indicate spatially neutral cues. Error bars indicate standard error of the mean. (Color figure online)

\section{Discussion}

The results of feature-validity effects for this experiment differed from those of Experiment 2, as there was no main effect of feature validity. (In Experiment 2, feature-valid trials were $\sim 2 \%$ more accurate than feature-invalid trials). The Bayesian analysis indicated this effect was inconclusive, but supported the lack of interaction with ISI.

\section{Experiment 4}

In Experiment 3, we did not find a significant feature-based effect. The absence of robust exogenous FBA may be because the temporal and spatial parameters used in our three experiments are not optimal to measure exogenous FBA influences. Thus, in Experiment 4, we decided to alter temporal and spatial parameters of the task. First, because a central goal of this study was to study the temporal dynamics of exogenous FBA, we decided to use a different set of ISIs that would capture the influence of the precue across a longer time scale: instead of $100 \mathrm{~ms}, 200 \mathrm{~ms}$, and $300 \mathrm{~ms}$, we used $100 \mathrm{~ms}, 300 \mathrm{~ms}$, and $500 \mathrm{~ms}$. Notably, $500 \mathrm{~ms}$ is about the time scale of endogenous FBA for some visual feature dimensions (Liu et al., 2007b). Second, to reduce spatial uncertainty, we changed the number of stimulus locations from eight to four, only presenting stimuli along the cardinal axes (i.e., the horizontal and vertical meridians). This made the stimulus display and search space much more consistent, as all four locations would contain either the target or a distractor on every trial. Furthermore, we explored whether the FBA effect would be more likely to emerge at some of the isoeccentric locations for which discriminability differs. Performance varies across isoeccentric locations at different polar angles (Abrams \& Carrasco, 2010; Abrams, Nizam, \& Carrasco, 2012; Carrasco, Talgar, $\&$ Cameron, 2001), with higher performance along the horizontal meridian than the vertical meridian - an effect known as the horizontal vertical anisotropy (HVA) - and higher performance along the lower rather than the upper vertical meridian - an effect known as the vertical meridian asymmetry (VMA). Thus, in this experiment we probed the locations that encompass the highest and the lowest visual ability.

Finally, we implemented a participant questionnaire at the very end of the experiment to gain insight into the participant's awareness of the cue, strategy in performing the task, and the potential influence of top-down strategy on the featurevalidity effect. A questionnaire was not used either in our previous experiments or in the previous studies using this task (Lin et al., 2011; Qian \& Liu, 2015). This questionnaire was motivated by the fact that there was no significant interaction between feature validity and ISI, as would be expected for transient effects of precues. Moreover, we wanted to assess whether some participants believed the cue indicated the color 
of the target and thus biased their strategy in performing the task, regardless of ISI or cue validity. Such an effect would not be bottom-up, stimulus-driven - and thus would not reflect exogenous FBA.

\section{Method}

\section{Participants}

Fifteen participants (nine female) with normal or corrected-tonormal vision were recruited to participate in the experiment. All observers were naïve to the purposes of the study, had normal color vision, and had normal or corrected-to-normal vision (glasses or contacts). The NYU Institutional Review Board approved this study.

\section{Apparatus}

The apparatus was the same as in Experiments 1-3.

\section{Stimuli and procedure}

Stimuli and procedure were the same as in Experiment 3, except that there were only four stimuli, which appeared along cardinal axes (upper and lower vertical meridian, and left and right horizontal meridian; see Fig. 7). In each trial, colors of the target and distractors were selected without replacement from the same pool of six colors used in previous experiments.

\section{Participant questionnaire}

Immediately upon completion of the second and last session, we asked all participants to complete a 9-item questionnaire regarding the experiment that probed what they believed they were being tested on, and any strategy or expectations they had while participating in the experiment. They were instructed to complete each question in order and to not go back to previous questions. The questions were as follows:

- Describe any strategy you had to perform the task.

- Describe what you believe the experiment was testing.

- Did you notice anything occurring on the screen in addition to what was included in the instructions (e.g., besides the target display and the "Respond!" text at the center)? Please be descriptive.

- What was your impression of the timing or pace of the task? Fast vs. slow? Did it vary, or did it seem consistent across blocks/trials?

- Do you believe your performance was affected by the location of the target (oval)? Or that it was consistent no matter the location?

- Do you believe your performance was affected by the color of the target? Did you get the impression that your performance was better or worse with a particular color? Please be specific.

- You may have noticed that there was a colored square that appeared at the center of the screen. Did you notice this stimulus (Yes or No)? If so, did you change your strategy depending on its color (Yes or No)? Please describe how this color square influenced your performance or strategy, if at all.

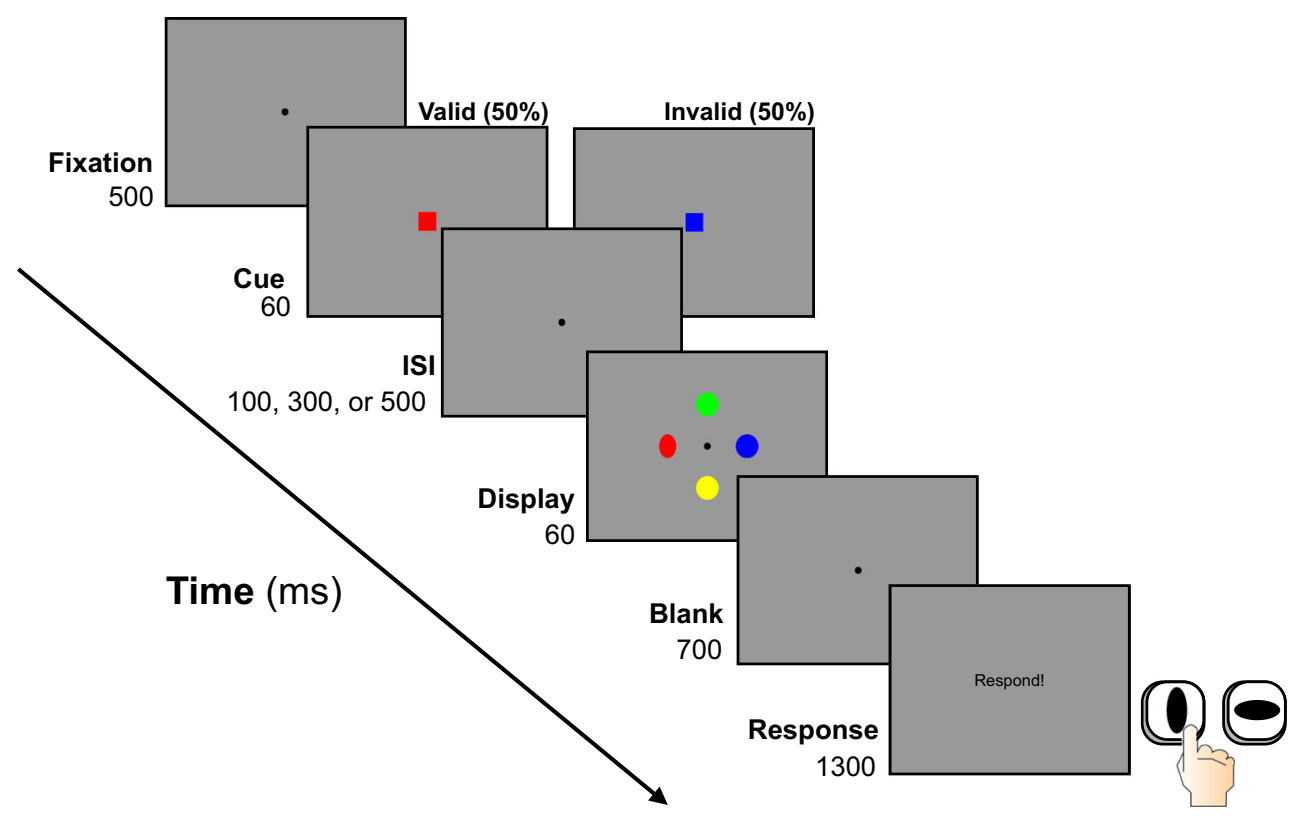

Fig. 7 Trial sequence for Experiment 4 
- Specifically, did the color of the square affect which stimulus you focused on or believed was the target oval (Yes or No)?

- Please provide any additional information about your experience completing the task, your strategies for optimizing performance, and anything you thought was unordinary or important.

\section{Results}

Accuracy and RT are shown in Fig. 8, separated by ISI and feature-validity conditions. We conducted a two-way (Feature Validity $\times$ ISI) analysis of variance (ANOVA) on accuracy. There was no two-way interaction, GreenhouseGeisser correction, $F(1,27.610)<1$. There was neither a main effect of ISI, Greenhouse-Geisser correction, $F(1$, $26.901)=1.234, p=.306$, nor a main effect of feature validity, $F(1,14)=2.587, p=.130$ ] Given the horizontalvertical anisotropy (HVA) effect (Abrams et al., 2012; Carrasco et al., 2001), we also conducted a three-way ANOVA that included axis (vertical vs. horizontal) in addition to feature validity and ISI, which revealed a significant main effect of axis, $F(1,14)=7.801, p<.02$, due to higher accuracy in trials on which the target appeared on the horizontal $(75.72 \%)$ compared with the vertical axis

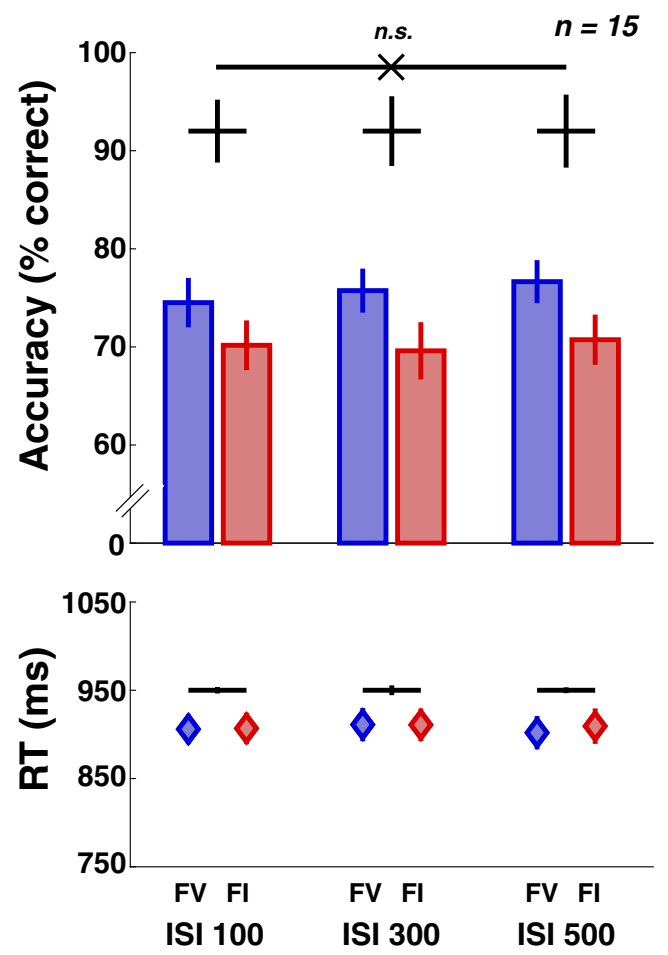

Fig. 8 Mean accuracy and median RT (correct trials) from Experiment 4. Blue bars and points indicate feature-valid trials; red indicates featureinvalid trials. Blue and red pairs show data for each ISI condition. Error bars indicate standard error of the mean. (Color figure online)
$(70.10 \%)$. There were no other significant main effects or interactions. We analyzed RT and verified that there was no speed-accuracy trade-off.

To investigate the influence of top-down factors on performance, especially relating to the feature precue, we divided participants into three groups according to their answers to the questionnaire administered upon completion of the final session. Specifically, we focused on the Questions 7 and 8, as these assessed whether participants were aware of the central precue and if the color of the precue influenced their belief of which of the peripheral shapes was the target oval. As a result, there were three distinct groups based on their knowledge and strategy regarding the precue: Strategy 1: Participants who noticed the presence of the precue and believed it indicated the color of the target $(n=3)$; Strategy 2: Participants who noticed the precue, but did not believe its color necessarily corresponded to that of the target $(n=4)$; Strategy 3: Participants who did not notice the presence of the precue and did not believe it indicated the color of the target $(n=8)$. Performance according to these strategy groups is plotted in Fig. 9. We conducted an ANOVA with ISI and feature validity as within-subjects factors, and strategy group $(1,2$, or 3$)$ as a between-subjects factor. This revealed a significant interaction between feature validity and strategy group, $F(1,1)=25.395 p<.001$, as well as a main effect of feature validity, $F(1,1)=27.563$, $p<.001$. The significant interaction was driven by the fact that Strategy Group 1, those who both noticed the cue and believed it indicated the color of the upcoming target oval, had higher accuracy in feature-valid trials $(87.37 \%)$ versus invalid trials $(58.04 \%)$. Another threeway ANOVA excluding these three observers revealed no significant interactions or main effects (all $p \mathrm{~s}>0.2$ ).

Again, we conducted a Bayesian model selection analysis. For the full data set (i.e., participants from Strategy Groups 1,2 , and $3 ; n=15$ ), there was positive evidence for the null effect of the ISI $\times$ Feature Validity interaction $-p \mathrm{BIC}(\mathrm{H} 1 \mid \mathrm{Data})=0.1006$ - and the ISI main effect $-p \mathrm{BIC}(\mathrm{H} 1 \mid$ Data $)=0.1227$. The main effect of feature validity was inconclusive $-p \mathrm{BIC}(\mathrm{H} 1 \mid$ Data $)=0.4795$. We repeated this analysis using only participants from Strategy Groups 2 and $3(n=12)$-that is, those who did not believe the precue's color matched the target's color. For this subset of observers, the interaction and both main effects showed positive evidence for the null hypothesis: ISI $\times$ Feature interaction $-p \mathrm{BIC}(\mathrm{H} 1 \mid$ Data $)=$ 0.0979; ISI main effect $-p \mathrm{BIC}(\mathrm{H} 1 \mid$ Data $)=0.16431$; feature validity main effect $-p \mathrm{BIC}(\mathrm{H} 1 \mid$ Data $)=0.2380$. These results indicate that when participants do not actively use the feature information to choose which stimulus to report, there is no effect of feature validity on performance - that is, there is no exogenous FBA effect. 


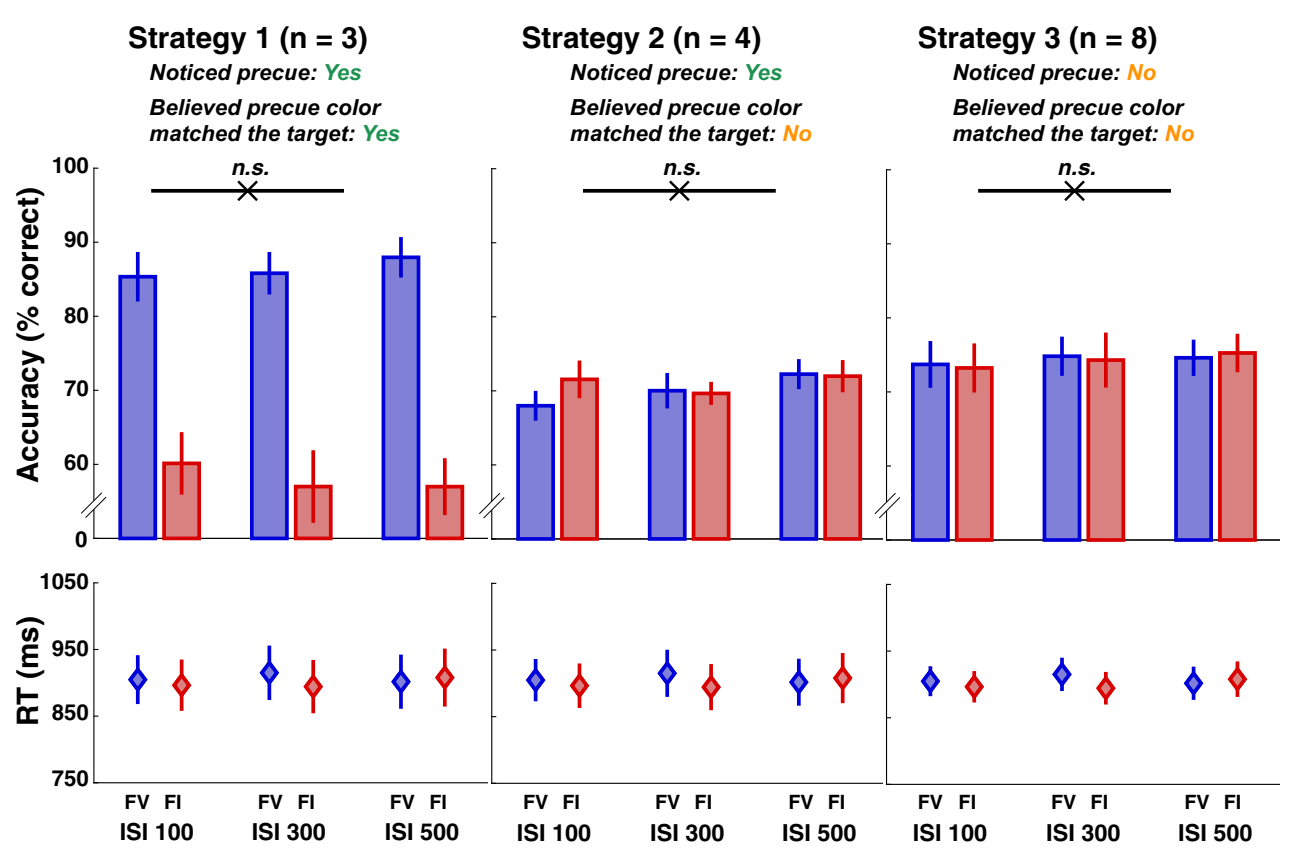

Fig. 9 Mean accuracy and median RT from Experiment 3. Separate panels indicate subgroups of subjects based on their reported awareness of the cue and their strategic use of the cue. Blue bars and points represent feature-valid trials, while red represents feature-invalid trials. Solid bars

\section{Discussion}

Again, we did not find a significant effect of the precue's feature validity on performance overall. There was a significant effect of the target location, consistent with the horizontal-vertical anisotropy (HVA) effect (Abrams et al., 2012; Carrasco et al., 2001). However, there was no influence of axis on the precue's effect on performance.

The participant questionnaire administered at the end of each participant's last session allowed us to assess the effects of exogenous FBA precues based on participants' reported knowledge and strategy regarding the precue. Interestingly, more than half ( 8 out of 15) of participants did not notice the precue at all, and therefore did not have any belief or strategy regarding the relation between the precue's color and that of the target. Further, participants who noticed the precue but did not believe its color to be indicative of the target exhibited no difference in performance between feature-valid and feature-invalid trials, suggesting that awareness of the cue alone did not result in any detectable effect on performance.

A feature-validity effect emerged only for those three participants who both noticed the precue and believed that it indicated the color of the target (Strategy Group 1,n=3). Strikingly, this strong and consistent feature-validity effect was present across all ISIs for this group, as quickly as 100 ms. This indicates that the effect is not an endogenous FBA effect, which takes at least $500 \mathrm{~ms}$ to be deployed (Liu et al., 2007b). Rather, this group of participants seem to have used and points indicate spatial-valid (SV) trials, while hollow bars and points indicate spatial-invalid (SI) trials. Each red and blue pair show data for each ISI condition within each group. Error bars indicate standard error of the mean. (Color figure online)

the precue's color to determine which target they would report the orientation of, leading to high accuracy in the featurevalid condition - when they reported the perceived orientation of the target ellipse - and very low accuracy in the feature-invalid condition - when they reported the perceived "orientation" of the circular distractor. Therefore, the topdown strategy that we infer was used by Strategy Group 1 is not endogenous FBA, in which participants voluntarily attend to the precued color before the target appears, but rather a later selection strategy, in which participants choose to report the orientation of the stimulus that shared the color of the precue - after both the precue and targets/distractor displays appeared. Excluding this small subgroup (1/5 of participants) from the analysis revealed no feature-validity effect. The inconclusive evidence for a feature-validity effect across all participants originated from only those who explicitly employed a biased search strategy based on the precue's color, whereas the remaining $4 / 5$ of participants showed no relation between precue validity and performance. Interestingly, about half of participants did not notice the precue. This is consistent with reports that participants often do not consciously perceive an exogenous spatial precue. Notably, behavioral effects of exogenous attention are found regardless of conscious awareness of a precue (e.g., McCormick, 1997).

In sum, these results show (1) no evidence for a bottom-up, automatic influence of the feature precue on performance, and (2) a top-down bias effect only for the few participants who believed the precue always indicated the identity or color of the target. 


\section{General discussion}

In four experiments with a total of 58 participants, we assessed the effect of a precue's color on an orientation judgment of a colored target among distractors. Several versions of a task based on two previous reports of exogenous FBA effects (Lin et al., 2011; Qian \& Liu, 2015) resulted in no significant influence of the precue's feature validity (i.e., whether its color matched the target's or a distractor's). This was the case (1) whether accuracy or RT was prioritized as the main measure, (2) with precues presented peripherally or centrally, (3) with cue-to-stimulus ISIs of varying durations, (4) with four or eight possible target locations, (5) at different meridians, (6) with either brief or long stimulus presentations, (7) and with either fixation-contingent or noncontingent stimulus displays. Notably, we found a strong effect of the precue's spatial validity when it appeared in the periphery with the 100-ms ISI, suitable for exogenous SA (Experiment 2). This indicates that task performance was sensitive to attentional manipulation, and that it was possible, in principle, to find an exogenous FBA effect. Importantly, we did not find a significant feature validity effect regardless of spatial validity — valid or invalid (peripheral; Experiments 1-2), or neutral (central; Experiments 3-4) - thus ruling out a possible confound of interaction with spatial attention.

Our inclusion of a Bayesian model selection analysis helped establish some key features of our data sets. In all four experiments, the main effect of feature validity was inconclusive, and the interaction with ISI and feature validity supported the null hypothesis. In Experiment 4, removing participants who, according to our questionnaire, employed a top-down feature-biased strategy revealed that there was no influence of the feature precue on performance when no top-down strategy was used; Bayes analysis confirmed this result. Taking these experiments together, we conclude that a bottom-up influence of visual feature information on perceptual performance - exogenous FBA - seems elusive at best.

The inclusion of the participant questionnaire in Experiment 4 shed light on how different participants performed the task. Further, it suggested that the lack of FBA effects in previous experiments, accompanied by inconclusive evidence from Bayesian analysis, could have been due to a similar top-down strategic bias employed by a small subset of participants who believed the precue was always valid regarding the feature of the target. Importantly, for the small subset of participants (1/5 of the total sample) in Experiment 4 who believed the cue indicated which stimulus was the target, the effect of feature validity was comparable across a wide ISI range $-100 \mathrm{~ms}, 300 \mathrm{~ms}$, and $500 \mathrm{~ms}$. Therefore, this is not an endogenous FBA effect, as endogenous FBA takes at least $500 \mathrm{~ms}$ to be deployed (Liu et al., 2007b). Rather, this small group of participants apparently considered that the precue's color was relevant to the task at hand, using it as a response cue to determine which stimulus's orientation they should report. As a result, those participants had high accuracy in the feature-valid condition - when they reported the perceived orientation of the target ellipse - and very low accuracy in the feature-invalid condition - when they reported the perceived "orientation" of the circular distractor. Therefore, the topdown strategy that we infer is responsible for these results in Experiment 4 is not endogenous FBA, in which participants voluntarily attend to the precued color before the target appears, but rather a later selection strategy, in which participants choose to report the orientation of the stimulus that shared the color of the precue.

As mentioned in the introduction, some have proposed that all reports of FBA, including endogenous, merely reflect bottom-up priming effects (Awh et al., 2012; Theeuwes, 2013). Specifically, they point out that FBA is usually discussed it in terms of an endogenous, top-down mechanism, then they review some studies of FBA that cannot rule out bottom-up priming, and conclude by asserting that it is likely that all studies that investigate FBA suffer from such a confound. Crucially, this hypothesis is in conflict with our current findings, in addition to being limited in its ability to explain a wide range of findings in the field whose designs and findings rule out the possibility of priming (e.g., Liu et al., 2011; Liu et al., 2007a; Liu, Slotnick, Serences, \& Yantis, 2003a; Liu et al., 2007b; Serences \& Yantis, 2007; White \& Carrasco, 2011; White et al., 2013, 2015). More recently, however, these authors have acknowledged instances of endogenous FBA that are not explained by bottom-up priming (Belopolsky \& Awh, 2016), or at least implicitly accepted that endogenous FBA can be top down (van Es et al., 2018). Had we found an effect of bottom-up, exogenous FBA precues, a discussion about its relation to priming would have been appropriate and perhaps necessary. However, given that we found no effect of the exogenous FBA precues, there is no reason to discuss these results in terms of the speculation that FBA is entirely bottom-up priming.

Our design and approach was inspired primarily by two previous studies (Lin et al., 2011; Qian \& Liu, 2015) that reported exogenous FBA effects on RT, with no indication of its effect on accuracy or its temporal dynamics. Another study reported a bottom-up effect of feature validity in a motion-direction change-detection task (Busse, Katzner, \& Treue, 2006). Specifically, faster RT only when a precue was both spatially valid and feature valid, and only at cue-to-target ISI time bin of 0-200 ms. The interpretation of these results, however, are hard to interpret for several reasons: (1) RT is the primary behavioral measure (see limits to this approach in the introduction). Moreover, the "go/no-go" detection task used is associated with pronounced criterion effects (e.g., Dang, Figueroa, \& Helton, 2018; Doneva \& De Fockert, 2014; Helton, Head, \& Russell, 2011; Helton, Weil, Middlemiss, \& Sawers, 2010), and the authors themselves conclude that 
they are unable to distinguish whether their results are due to changes in sensitivity or criterion (p. 2026). (2) In the targetpresent trials - $75 \%$ of trials - of Experiment 2, in which the significant RT feature validity effect was found, detection accuracy was near chance $-76.9 \%$. Thus, the RT results are uninterpretable. Further, because the authors do not report accuracy for each of the cueing conditions (two spatial validities by two feature validities), the RT results could reflect speed-accuracy trade-offs (Pachella, 1973). (3) The study assessed a wide range of ISIs, but reported performance for long ISI bins - spanning $200 \mathrm{~ms}$ each. The only significant effect, found in the shortest bin (0-200 ms), could be driven by either a precue or a simultaneous cue. (4) The cue consisted of a color change in the moving dots that comprised the target; thus, it is unclear if a behavioral effect of feature validity would represent bottom-up FBA, or a form of attentional capture to the stimulus itself. These four factors challenge the interpretation of the results reported in that study.

We aimed to replicate the effects of exogenous FBA to investigate the temporal dynamics of such effects. Had we found effects of exogenous FBA we had planned to investigate its interaction with exogenous spatial attention, as we have done for their endogenous counterparts (White et al., 2015). Moreover, we viewed exogenous FBA as a potential tool for affecting perceptual learning given our recent findings of covert attention on perceptual learning: Exogenous SA enables perceptual learning (Szpiro \& Carrasco, 2015) and facilitates location transfer (Donovan et al., 2015); endogenous SA also facilitates location transfer (Donovan \& Carrasco, 2018); and endogenous FBA facilitates recovery of visual function in the blind field of cortically blind patients (Cavanaugh, Barbot, Carrasco, \& Huxlin, 2017). Exogenous attention is particularly promising for rehabilitation efforts, as it requires no additional cognitive effort by the participants, which is a great asset for long-term clinical training regimes. Alas, given the elusive-at-best effect of exogenous FBA with the cueing protocol used in the studies reporting exogenous FBA (Lin et al., 2011; Qian \& Liu, 2015), it now seems unpromising to pursue this line of research, and investigating its possible role in visual search may be unwarranted. Nevertheless, we consider it important to report these findings so that the results reported in those previous studies are taken with caution. Even though a cueing protocol has also been used in most studies of endogenous FBA, the possibility remains that the existence of exogenous FBA could be uncovered with other experimental protocols.

Acknowledgements This research was funded by NIH-NEI-ROIEY16200 and NIH-NEI-R01-EY019693. We thank the members of the Carrasco lab for their useful comments for this research and manuscript.

Open practices statement This study was not preregistered. The data will be available upon request.

\section{References}

Abrams, J., \& Carrasco, M. (2010). Visual performance fields in noise. Journal of Vision, 10(7). doi:https://doi.org/10.1167/10.7.1367

Abrams, J., Nizam, A., \& Carrasco, M. (2012). Isoeccentric locations are not equivalent: The extent of the vertical meridian asymmetry. Vision Research, 52(1), 70-78. doi:https://doi.org/10.1016/j.visres. 2011.10.016

Adamian, N., Slaustaite, E., \& Andersen, S. K. (2019). Top-down attention is limited within but not between feature dimensions. Journal of Cognitive Neuroscience, 31(8), 1173-1183. doi:https://doi.org/10. 1162/jocn_a_01383

Andersen, S. K., Fuchs, S., \& Müller, M. (2011). Effects of featureselective and spatial attention at different stages of visual processing. Journal of Ccognitive Neuroscience, 23(1), 238-246. doi:https:// doi.org/10.1162/jocn.2009.21328

Andersen, S. K., Hillyard, S. A., \& Müller, M. M. (2013). Global facilitation of attended features is obligatory and restricts divided attention. The Journal of neuroscience: The Official Journal of the Society for Neuroscience, 33(46), 18200-18207. doi:https://doi. org/10.1523/JNEUROSCI.1913-13.2013

Awh, E., Belopolsky, A. V., \& Theeuwes, J. (2012). Top-down versus bottom-up attentional control: A failed theoretical dichotomy. Trends in Cognitive Sciences, 16(8), 437-443. doi:https://doi.org/ 10.1016/j.tics.2012.06.010

Barbot, A., \& Carrasco, M. (2017). Attention modifies spatial resolution according to task demands. Psychological Science. doi:https://doi. org/10.1177/0956797616679634

Barbot, A., Landy, M. S., \& Carrasco, M. (2012). Differential effects of exogenous and endogenous attention on second-order texture contrast sensitivity. Journal of Vision, 12(8). doi:https://doi.org/10. $1167 / 12 / 8 / 6$

Belopolsky, A. V., \& Awh, E. (2016). The role of context in volitional control of feature-based attention. Journal of Experimental Psychology: Human Perception and Performance, 42(2), 213224. doi:https://doi.org/10.1037/xhp0000135

Brainard, D. H. (1997). The Psychophysics Toolbox. Spatial vision, 10(4), 433-436. Retrieved from http://www.ncbi.nlm.nih.gov/ pubmed/9176952

Busse, L., Katzner, S., \& Treue, S. (2006). Spatial and feature-based effects of exogenous cueing on visual motion processing. Vision Research, 46(13), 2019-2027.

Cameron, E. L., Tai, J. C., Eckstein, M. P., \& Carrasco, M. (2004). Signal detection theory applied to three visual search tasks-Identification, yes/no detection and localization. Spatial Vision, 17(4/5), 295-325. Retrieved from http://www.ncbi.nlm.nih.gov/entrez/query.fcgi? $\mathrm{cmd}=$ Retrieve $\& \mathrm{db}=$ PubMed\&dopt=Citation\&list_uids $=15559107$

Carrasco, M. (2011). Visual attention: The past 25 years. Vision Research, 51(13), 1484-1525. doi:https://doi.org/10.1016/j.visres.2011.04. 012

Carrasco, M. (2014). Spatial attention: Perceptual modulation. In S. Kastner \& A. C. Nobre (Eds.), The Oxford handbook of attention (pp. 183-230). Oxford, UK: Oxford University Press.

Carrasco, M., \& Barbot, A. (2015). How attention affects spatial resolution. Cold Spring Harbor Symposia on Quantitative Biology, 79, 149-160. doi:https://doi.org/10.1101/sqb.2014.79.024687

Carrasco, M., Giordano, A. M., \& McElree, B. (2004a). Temporal performance fields: Visual and attentional factors. Vision Research, 44(12), 1351-1365. doi: https://doi.org/10.1016/j.visres.2003.11. 026

Carrasco, M., Giordano, A. M., \& McElree, B. (2006). Attention speeds processing across eccentricity: Feature and conjunction searches. Vision Research, 46(13), 2028-2040. doi:https://doi.org/10.1016/j. visres.2005.12.015 
Carrasco, M., Ling, S., \& Read, S. (2004b). Attention alters appearance. Nature Neuroscience, 7(3), 308-313. doi: https://doi.org/10.1038/ $\mathrm{nn} 1194$

Carrasco, M., \& McElree, B. (2001). Covert attention accelerates the rate of visual information processing. Proceedings of the National Academy of Sciences of the United States of America, 98(9), 5363-5367. doi:https://doi.org/10.1073/pnas.081074098

Carrasco, M., Ponte, D., Rechea, C., \& Sampedro, M. J. (1998). "Transient structures": The effects of practice and distractor grouping on within-dimension conjunction searches. Percept and Psychophys, 60(7), 1243-1258. Retrieved from http://www.ncbi. nlm.nih.gov/entrez/query.fcgi?cmd=Retrieve $\& \mathrm{db}=\mathrm{PubMed} \& \mathrm{dopt}=$ Citation\&list uids $=9821785$

Carrasco, M., Talgar, C. P., \& Cameron, E. L. (2001). Characterizing visual performance fields: Effects of transient covert attention, spatial frequency, eccentricity, task and set size. Spatial Vision, 15(1), 61-75. Retrieved from http://www.ncbi.nlm.nih.gov/entrez/query. fcgi? $\mathrm{cmd}=$ Retrieve $\& \mathrm{db}=$ PubMed $\& \mathrm{dopt}=$ Citation $\&$ list uids $=$ 11893125

Cavanaugh, M. R., Barbot, A., Carrasco, M., \& Huxlin, K. R. (2017). Feature-based attention potentiates recovery of fine direction discrimination in cortically blind patients. Neuropsychologia. doi: https://doi.org/10.1016/j.neuropsychologia.2017.12.010

Cavanaugh, M. R., Barbot, A., Carrasco, M., \& Huxlin, K. R. (2019). Feature-based attention potentiates recovery of fine direction discrimination in cortically blind patients. Neuropsychologia, 128, 315-324.

Cheal, M., \& Lyon, D. R. (1991a). Central and peripheral precuing of forced-choice discrimination. Quarterly Journal of Experimental Psychology A, 43(4), 859-880. Retrieved from http://www.ncbi. nlm.nih.gov/entrez/query.fcgi? $\mathrm{cmd}=$ Retrieve $\& \mathrm{db}=\mathrm{PubMed} \& \mathrm{dopt}=$ Citation\&list uids $=1775667$

Cheal, M., \& Lyon, D. R. (1991b). Importance of precue location in directing attention. Acta Psychologica, 76(3), 201-211. Retrieved from http://www.ncbi.nlm.nih.gov/entrez/query.fcgi? $\mathrm{cmd}=$ Retrieve $\& \mathrm{db}=\mathrm{PubMed} \& \mathrm{dopt}=$ Citation \&list uids $=1927574$

Cheal, M., Lyon, D. R., \& Hubbard, D. C. (1991). Does attention have different effects on line orientation and line arrangement discrimination? Quarterly Journal of Experimental Psychology A, 43(4), 825-857. Retrieved from http://www.ncbi.nlm.nih.gov/entrez/ query.fcgi? $\mathrm{cmd}=$ Retrieve $\& \mathrm{db}=\mathrm{PubMed} \& \mathrm{dopt}=$ Citation\&list_uids $=$ 1775666

Corbetta, M., \& Shulman, G. L. (2002). Control of goal-directed and stimulus-driven attention in the brain. Nature Reviews Neuroscience, 3(3), 201-215. doi:https://doi.org/10.1038/nrn755

Dang, J. S., Figueroa, I. J., \& Helton, W. S. (2018). You are measuring the decision to be fast, not inattention: The sustained attention to response task does not measure sustained attention. Experimental Brain Research, 236(8), 2255-2262.

Doneva, S. P., \& De Fockert, J. W. (2014). More conservative go/no-go response criterion under high working memory load. Journal of Cognitive Psychology, 26(1), 110-117.

Donovan, I., \& Carrasco, M. (2018). Endogenous spatial attention during perceptual learning facilitates location transfer. Journal of Vision, 18(11), 7-7. doi:https://doi.org/10.1167/18.11.7

Donovan, I., Szpiro, S., \& Carrasco, M. (2015). Exogenous attention facilitates location transfer of perceptual learning. Journal of Vision, 15(10), 11. doi:https://doi.org/10.1167/15.10.11

Dugué, L., Merriam, E. P., Heeger, D. J., \& Carrasco, M. (2017). Specific visual sub-regions of TPJ mediate reorienting of spatial attention. Cereb Cortex, 28(7), 2375-2390. doi:https://doi.org/10.1093/ cercor/bhx140

Eckstein, M. P. (2011). Visual search: A retrospective. Journal of Vision, $11(5), 14$

Egeth, H. E., Virzi, R. A., \& Garbart, H. (1984). Searching for conjunctively defined targets. Journal of Experimental Psychology: Human
Perception and Performance, 10(1), 32-39. Retrieved from http:// www.ncbi.nlm.nih.gov/entrez/query.fcgi?cmd=Retrieve $\& d b=$ PubMed\&dopt $=$ Citation\&list uids $=6242762$

Eimer, M. (2014). The neural basis of attentional control in visual search. Trends in Cognitive Sciences, 18(10), 526-535. doi:https://doi.org/ 10.1016/j.tics.2014.05.005

Fang, M. W. H., Becker, M. W., \& Liu, T. (2019). Attention to colors induces surround suppression at category boundaries. Scientific Reports, 9(1), 1443. doi:https://doi.org/10.1038/s41598-01837610-7

Faul, F., Erdfelder, E., Lang, A.-G., \& Buchner, A. (2007). G* Power 3: A flexible statistical power analysis program for the social, behavioral, and biomedical sciences. Behavior Research Methods, 39(2), 175191.

Garcia-Perez, M. A. (1998). Forced-choice staircases with fixed step sizes: Asymptotic and small-sample properties. Vision Research, 38(12), 1861-1881. doi:https://doi.org/10.1016/S0042-6989(97) 00340-4

Giordano, A. M., McElree, B., \& Carrasco, M. (2009). On the automaticity and flexibility of covert attention: A speed-accuracy trade-off analysis. Journal of Vision, 9(3), 1-10. doi:https://doi.org/10.1167/ 9.3.30/9/3/30/

Grubb, M. A., Behrmann, M., Egan, R., Minshew, N. J., Carrasco, M., \& Heeger, D. J. (2013a). Endogenous spatial attention: evidence for intact functioning in adults with autism. Autism Res, 6(2), 108-118. doi:https://doi.org/10.1002/aur.1269

Grubb, M. A., Behrmann, M., Egan, R., Minshew, N. J., Heeger, D. J., \& Carrasco, M. (2013b). Exogenous spatial attention: Evidence for intact functioning in adults with autism spectrum disorder. Journal of Vision, 13(14). doi:https://doi.org/10.1167/13.14.9

Hayden, B. Y., \& Gallant, J. L. (2009). Combined effects of spatial and feature-based attention on responses of V4 neurons. Vision Resrch, 49(10), 1182-1187. doi:https://doi.org/10.1016/j.visres.2008.06. 011

Hein, E., Rolke, B., \& Ulrich, R. (2006). Visual attention and temporal discrimination: Differential effects of automatic and voluntary cueing. Visual Cogntion, 13(1), 29-50.

Helton, W. S., Head, J., \& Russell, P. N. (2011). Reliable-and unreliablewarning cues in the sustained attention to response task. Experimental Brain Research, 209(3), 401-407.

Helton, W. S., Weil, L., Middlemiss, A., \& Sawers, A. (2010). Global interference and spatial uncertainty in the sustained attention to response task (SART). Consciousness and Cognition, 19(1), 77-85.

Herrmann, K., Heeger, D. J., \& Carrasco, M. (2012). Feature-based attention enhances performance by increasing response gain. Vision Research, 74, 10-20. doi:https://doi.org/10.1016/j.visres.2012.04. 016

Jigo, M., \& Carrasco, M. (2018). Attention alters spatial resolution by modulating second-order processing. Journal of Vision, 18(7), 2-2. doi:https://doi.org/10.1167/18.7.2

Jigo, M., Gong, M., \& Liu, T. (2018). Neural determinants of task performance during feature-based attention in human cortex. eNeuro, 5(1). doi:https://doi.org/10.1523/ENEURO.0375-17.2018

Katzner, S., Busse, L., \& Treue, S. (2009). Attention to the color of a moving stimulus modulates motion-signal processing in macaque area MT: Evidence for a unified attentional system. Frontiers in Systems Neuroscience, 3, 12. Retrieved from https://www. frontiersin.org/article/10.3389/neuro.06.012.2009

Lin, J. Y., Hubert-Wallander, B., Murray, S. O., \& Boynton, G. M. (2011). Rapid and reflexive feature-based attention. Journal of Vision, 11(12), 12-12. doi:https://doi.org/10.1167/11.12.12

Ling, S., \& Carrasco, M. (2006). Sustained and transient covert attention enhance the signal via different contrast response functions. Vision Res, 46(8-9), 1210-1220. Retrieved from http://www.ncbi.nlm.nih. gov/entrez/query.fcgi?cmd=Retrieve $\& \mathrm{db}=$ PubMed $\& \mathrm{dopt}=$ Citation\&list uids $=16005931$ 
Ling, S., \& Carrasco, M. (2007). Transient covert attention does alter appearance: A reply to Schneider (2006). Perception \& Psychophysics, 69(6), 1051-1058. Retrieved from http://www. ncbi.nlm.nih.gov/entrez/query.fcgi? cmd=Retrieve $\& \mathrm{db}=$ PubMed\&dopt=Citation\&list_uids $=18018987$

Ling, S., Liu, T., \& Carrasco, M. (2009). How spatial and feature-based attention affect the gain and tuning of population responses. Vision Research, 49(10), 1194-1204. doi:https://doi.org/10.1016/j.visres. 2008.05.025

Liu, T., Becker, M. W., \& Jigo, M. (2013). Limited featured-based attention to multiple features. Vision Research, 85, 36-44. doi:https://doi. org/10.1016/j.visres.2012.09.001

Liu, T., Hospadaruk, L., Zhu, D. C., \& Gardner, J. L. (2011). Featurespecific attentional priority signals in human cortex. The Journal of Neuroscience, 31(12), 4484. Retrieved from http://www.jneurosci. org/content/31/12/4484.abstract

Liu, T., \& Jigo, M. (2017). Limits in feature-based attention to multiple colors. Attention, Perception, \& Psychophysics, 79(8), 2327-2337. doi:https://doi.org/10.3758/s13414-017-1390-x

Liu, T., Larsson, J., \& Carrasco, M. (2007a). Feature-based attention modulates orientation-selective responses in human visual cortex. Neuron, 55(2), 313-323. doi:https://doi.org/10.1016/j.neuron. 2007.06.030

Liu, T., \& Mance, I. (2011). Constant spread of feature-based attention across the visual field. Vision Research, 51(1), 26-33. doi:https:// doi.org/10.1016/j.visres.2010.09.023

Liu, T., Slotnick, S. D., Serences, J. T., \& Yantis, S. (2003a). Cortical mechanisms of feature-based attentional control. Cerebral Cortex, 13(12), 1334-1343. doi:https://doi.org/10.1093/cercor/bhg080

Liu, T., Slotnick, S. D., Serences, J. T., \& Yantis, S. (2003b). Cortical mechanisms of feature-based attentional control. Cerebral Cortex, 13(12), 1334-1343. Retrieved from http://www.ncbi.nlm.nih.gov/ entrez/query.fcgi? cmd $=$ Retrieve $\& d b=$ PubMed $\& d o p t=$ Citation\&list uids $=14615298$

Liu, T., Stevens, S. T., \& Carrasco, M. (2007b). Comparing the time course and efficacy of spatial and feature-based attention. Vision Research, 47(1), 108-113. doi:https://doi.org/10.1016/j.visres. 2006.09.017

Masson, M. E. J. (2011). A tutorial on a practical Bayesian alternative to null-hypothesis significance testing. Behavior Research Methods, 43(3), 679-690. doi:https://doi.org/10.3758/s13428-010-0049-5

McCormick, P. A. (1997). Orienting attention without awareness. Journal of Experimental Psychology: Human Perception and Performance, 23(1), 168.

McElree, B., \& Carrasco, M. (1999). The temporal dynamics of visual search: evidence for parallel processing in feature and conjunction searches. Journal of experimental psychology Human perception and performance, 25(6), 1517-1539. Retrieved from http://www. ncbi.nlm.nih.gov/entrez/query.fcgi? cmd=Retrieve $\& d b=$ PubMed\&dopt=Citation\&list uids $=10641310$

McElree, B., \& Dosher, B. A. (1989). Serial position and set size in shortterm memory: the time course of recognition. Journal of Experimental Psychology: General, 118(4), 346.

McMains, S. A., Fehd, H. M., Emmanouil, T. A., \& Kastner, S. (2007). Mechanisms of feature- and space-based attention: Response modulation and baseline increases. Journal of Neurophysiology, 98(4), 2110-2121. doi:https://doi.org/10.1152/jn.00538.2007

Moore, C. M., \& Egeth, H. (1998). How does feature-based attention affect visual processing? Journal of Experimental Psychology: Human Perception and Performance, 24(4), 1296-1310. Retrieved from http://www.ncbi.nlm.nih.gov/entrez/query.fcgi? cmd $=$ Retrieve $\& d b=$ PubMed\&dopt=Citation\&list_uids=9706716

Müller, H. J., Heller, D., \& Ziegler, J. (1995). Visual search for singleton feature targets within and across feature dimensions. Perception \& Psychophysics, 57(1), 1-17. Retrieved from http://www.ncbi.nlm.
nih.gov/entrez/query.fcgi?cmd=Retrieve $\& \mathrm{db}=$ PubMed $\&$ dopt $=$ Citatiüon\&list uids $=7885801$

Müller, H. J., \& Rabbitt, P. M. (1989). Reflexive and voluntary orienting of visual attention: time course of activation and resistance to interruption. Journal of experimental psychology. Human perception and performance, 15, 315-330. Retrieved from http://www.ncbi. nlm.nih.gov/entrez/query.fcgi?cmd=Retrieve $\& \mathrm{db}=\mathrm{PubMed} \& \mathrm{dopt}=$ Citation\&list uids $=2525601$

Müller, M. (2014). Neural mechanisms of feature-based attention. In G. R. Mangun (Ed.), Cognitive electrophysiology of attention: Signals of the mind (pp. 123-35). San Diego, CA: Academic Press.

Nakayama, K., \& Mackeben, M. (1989). Sustained and transient components of focal visual attention. Vision Research, 29(11), 1631-1647. Retrieved from http://www.ncbi.nlm.nih.gov/entrez/query.fcgi? $\mathrm{cmd}=$ Retrieve $\& \mathrm{db}=$ PubMed\&dopt=Citation \&list uids $=2635486$

Nakayama, K., \& Martini, P. (2010). Situating visual search. Vision Research, 51(13), 1526-1537. doi:https://doi.org/10.1016/j.visres. 2010.09.003

Pachella, R. (1973). The interpretation of reaction time in information processing research (Technical Report). Retrieved from https://apps. dtic.mil/dtic/tr/fulltext/u2/787880.pdf

Patzwahl, D. R., \& Treue, S. (2009). Combining spatial and feature-based attention within the receptive field of MT neurons. Vision Research, 49(10), 1188-1193. doi:https://doi.org/10.1016/j.visres.2009.04. 003

Pelli, D. G. (1997). The VideoToolbox software for visual psychophysics: Transforming numbers into movies. Spatial Vision, 10(4), 437-442. Retrieved from http://www.ncbi.nlm.nih.gov/pubmed/9176953

Qian, C., \& Liu, T. (2015). Involuntary attention in the absence of visual awareness. Visual Cognition, 23(7), 840-843. doi:https://doi.org/10. 1080/13506285.2015.1093249

Raftery, A. E. (1995). Bayesian model selection in social research. Sociological Methodology, 25, 111-163. doi:https://doi.org/10. 2307/271063

Reed, A. V. (1973). Speed-accuracy trade-off in recognition memory. Science, 181(4099), 574. Retrieved from http://science. sciencemag.org/content/181/4099/574.abstract

Remington, R. W., Johnston, J. C., \& Yantis, S. (1992). Involuntary attentional capture by abrupt onsets. Perception \& Psychophysics, 51(3), 279-290. Retrieved from http://www.ncbi.nlm.nih.gov/ entrez/query.fcgi?cmd=Retrieve $\& \mathrm{db}=\mathrm{PubMed} \& \mathrm{dopt}=$ Citation\&list uids $=1561053$

Rossi, A. F., \& Paradiso, M. A. (1995). Feature-specific effects of selective visual attention. Vision Res, 35(5), 621-634.

Saenz, M., Buracas, G. T., \& Boynton, G. M. (2002). Global effects of feature-based attention in human visual cortex. Nature Neuroscience, 5, 631. doi:https://doi.org/10.1038/nn876

Schoenfeld, M. A., Hopf, J. M., Martinez, A., Mai, H. M., Sattler, C., Gasde, A., . . Hillyard, S. A. (2007). Spatio-temporal analysis of feature-based attention. Cerebral Cortex, 17(10), 2468-2477. doi: https://doi.org/10.1093/cercor/bhl154

Serences, J. T., \& Boynton, G. M. (2007). Feature-based attentional modulations in the absence of direct visual stimulation. Neuron, 55(2), 301-312. doi:https://doi.org/10.1016/j.neuron.2007.06.015

Serences, J. T., \& Kastner, S. (2014). A multi-level account of selective attention. In A. C. Nobre \& S. Kastner (Eds.), The Oxford handbook of attention. Oxford, UK: Oxford University Press.

Serences, J. T., \& Yantis, S. (2007). Spatially selective representations of voluntary and stimulus-driven attentional priority in human occipital, parietal, and frontal cortex. Cerebral Cortex, 17(2), 284-293. doi:https://doi.org/10.1093/cercor/bhj146

Shih, S. I., \& Sperling, G. (1996). Is there feature-based attentional selection in visual search? Journal of Experimental Psychology: Human Perception and Performance, 22(3), 758-779. Retrieved from http://www.ncbi.nlm.nih.gov/entrez/query.fcgi?cmd= Retrieve $\& \mathrm{db}=$ PubMed\&dopt $=$ Citation\&list_uids $=8666962$ 
Sohn, W., Chong, S. C., Papathomas, T. V., \& Vidnyánszky, Z. (2005). Cross-feature spread of global attentional modulation in human area MT+. Neuroreport, 16(12), 1389-1393.

Störmer, V. S., \& Alvarez, G. A. (2014). Feature-based attention elicits surround suppression in feature space. Current Biology, 24(17), 1985-1988. doi:https://doi.org/10.1016/j.cub.2014.07.030

Szpiro, S. F., \& Carrasco, M. (2015). Exogenous attention enables perceptual learning. Psychological Science. doi:https://doi.org/10.1177/ 0956797615598976

Theeuwes, J. (2013). Feature-based attention: it is all bottom-up priming. Philosophical Transactions of the Royal Society B: Biological Sciences, 368(1628), 20130055. doi:https://doi.org/10.1098/rstb. 2013.0055

Treisman, A. (1982). Perceptual grouping and attention in visual search for features and for objects. Journal of Experimental Psychology: Human Perception and Performance, 8(2), 194-214. doi:https://doi. org/10.1037/0096-1523.8.2.194

Treisman, A. (1988). Features and objects: the fourteenth Bartlett memorial lecture. Quarterly Journal of Experimental Psychology A, 40(2), 201-237. Retrieved from http://www.ncbi.nlm.nih.gov/entrez/ query.fcgi? $\mathrm{cmd}=$ Retrieve $\& \mathrm{db}=$ PubMed $\&$ dopt $=$ Citation $\&$ list uids $=$ 3406448

Treisman, A. M., \& Gelade, G. (1980). A feature-integration theory of attention. Cognitive Psychology, 12(1), 97-136. doi:https://doi.org/ 10.1016/0010-0285(80)90005-5

Treue, S. (2014). Object- and feature-based attention: Monkey physiology. In A. C. Nobre \& S. Kastner (Eds.), Oxford handbook of attention. New York, NY: Oxford University Press. doi:https://doi.org/ 10.1093/oxfordhb/9780199675111.013.008

Treue, S., \& Martínez-Trujillo, J. C. (1999). Feature-based attention influences motion processing gain in macaque visual cortex. Nature, 399(6736), 575-579. doi:https://doi.org/10.1038/21176

Treue, S., \& Martínez-Trujillo, J. C. (2007). Attending to features inside and outside the spotlight of attention. Neuron, 55(2), 174-176. doi: https://doi.org/10.1016/j.neuron.2007.07.005

van Es, D. M., Theeuwes, J., \& Knapen, T. (2018). Spatial sampling in human visual cortex is modulated by both spatial and feature-based attention. eLife, 7, e36928. doi:https://doi.org/10.7554/eLife.36928
Watson, A. B., \& Pelli, D. G. (1983). QUEST: A Bayesian adaptive psychometric method. Perception \& Psychophysics, 33(2), 113120. Retrieved from http://www.ncbi.nlm.nih.gov/entrez/query. fcgi? $\mathrm{cmd}=$ Retrieve $\& \mathrm{db}=$ PubMed $\& d o p t=$ Citation\&list_uids $=$ 6844102

White, A. L., \& Carrasco, M. (2011). Feature-based attention involuntarily and simultaneously improves visual performance across locations. Journal of Vision, 11(6). doi:https://doi.org/10.1167/11.6.15

White, A. L., Rolfs, M., \& Carrasco, M. (2013). Adaptive deployment of spatial and feature-based attention before saccades. Vision Research, 85, 26-35. doi:https://doi.org/10.1016/j.visres.2012.10.017

White, A. L., Rolfs, M., \& Carrasco, M. (2015). Stimulus competition mediates the joint effects of spatial and feature-based attention. Journal of Vision, 15(14), 7-7. doi:https://doi.org/10.1167/15.14.7

Wolfe, J. M., Butcher, S. J., Lee, C., \& Hyle, M. (2003). Changing your mind: On the contributions of top-down and bottom-up guidance in visual search for feature singletons. Journal of Experimental Psychology: Human Perception and Performance, 29(2), 483502. Retrieved from http://www.ncbi.nlm.nih.gov/entrez/query. fcgi? $\mathrm{cmd}=$ Retrieve $\& \mathrm{db}=$ PubMed $\&$ dopt $=$ Citation $\&$ list_uids $=$ 12760630

Wolfe, J. M., \& Horowitz, T. S. (2004). What attributes guide the deployment of visual attention and how do they do it? Nature Reviews Neuroscience, 5(6), 495-501. doi:https://doi.org/10.1038/ nrn1411nrn1411

Yeshurun, Y., \& Carrasco, M. (1998). Attention improves or impairs visual performance by enhancing spatial resolution. Nature, 396(6706), 72-75. Retrieved from http://www.ncbi.nlm.nih.gov/ entrez/query.fcgi cmd $=$ Retrieve $\& d b=$ PubMed $\& d o p t=$ Citation\&list_uids $=9817201$

Yeshurun, Y., Montagna, B., \& Carrasco, M. (2008). On the flexibility of sustained attention and its effects on a texture segmentation task. Vision Research, 48(1), 80-95. Retrieved from http://www.ncbi. nlm.nih.gov/entrez/query.fcgi?cmd=Retrieve $\& \mathrm{db}=\mathrm{PubMed} \& \mathrm{dopt}=$ Citation\&list_uids $=18076966$

Publisher's note Springer Nature remains neutral with regard to jurisdictional claims in published maps and institutional affiliations. 